\title{
Microscopic treatment of binary interactions in the nonequilibrium dynamics of partially Bose-condensed trapped gases
}

\author{
N. P. Proukakis and K. Burnett \\ Clarendon Laboratory, Department of Physics, University of Oxford, Oxford, OX1 3PU, United Kingdom \\ H. T. C. Stoof \\ Institute for Theoretical Physics, University of Utrecht, Princetonplein 5, P.O. Box 80.006, 3508 TA Utrecht, The Netherlands
}

(Received 21 March 1997)

\begin{abstract}
In this paper we use microscopic arguments to derive a nonlinear Schrödinger equation for trapped Bosecondensed gases. This is made possible by considering the equations of motion of various anomalous averages. The resulting equation explicitly includes the effect of repeated binary interactions (in particular ladders) between the atoms. Moreover, under the conditions where dressing of the intermediate states of a collision can be ignored, this equation is shown to reduce to the conventional Gross-Pitaevskii equation in the pseudopotential limit. Extending the treatment, we show first how the occupation of excited (bare particle) states affects the collisions, and thus obtain the many-body $T$-matrix approximation in a trap. In addition, we discuss how the bare particle many-body $T$ matrix gets dressed by mean fields due to condensed and excited atoms. We conclude that the most commonly used version of the Gross-Pitaevskii equation can only be put on a microscopic basis for a restrictive range of conditions. For partial condensation, we need to take account of interactions between condensed and excited atoms, which, in a consistent formulation, should also be expressed in terms of the many-body $T$ matrix. This can be achieved by considering fluctuations around the condensate mean field beyond those included in the conventional finite temperature mean field, i.e., Hartree-FockBogoliubov, theory. [S1050-2947(98)00302-3]

PACS number(s): 03.75.Fi, 05.30.Jp, 31.15.Ne
\end{abstract}

\section{INTRODUCTION}

The observation of Bose-Einstein condensation (BEC) in alkali atomic gases [1-3] strongly motivates a description of the evolution of the condensate that takes full account of the microscopic nature of atomic interactions in a trap, both close to and far from equilibrium. The conventional description relies heavily on the well-known Gross-Pitaevskii equation (GPE) [4], also known as the nonlinear Schrödinger equation. In this equation, one assumes that the atoms are all effectively condensed and the atomic interactions can be accurately modeled by a pseudopotential, expressed in terms of the $s$-wave scattering length. This theory appears to make good predictions about the condensate's properties [5-13] and it is obviously desirable to put this phenomenological theory on a clear microscopic basis. In fact, in spite of its frequent use, a direct link of this effective interaction approach to microscopic properties of the gas that includes a discussion of the effect of the mean fields on the intermediate states of a binary collision, appears to be lacking. In this paper we will address such issues in a derivation of a nonlinear Schrödinger equation based on these microscopic properties and what we believe are reasonable assumptions about them.

In the first part of the paper we shall deal with the weakly interacting case [14] and show how one can introduce an effective interaction that takes account of all possible repeated binary atomic collisions. This limit of weak interactions has been treated quite extensively in the literature. We note the work of Beliaev [15] who obtained an effective interaction by means of a diagrammatic perturbation expansion for the homogeneous gas. The replacement of the actual interatomic potential by an effective one has also been extensively discussed by Popov in the context of effective action path integrals [16]. Following the work by Huang and Yang [17] and Lee, Huang, and Yang [18], this effective interaction is often expressed in the usual dilute Bose gas pseudopotential form. In this paper, we will discuss the issue of an effective potential by means of an alternative approach, somewhat similar to Beliaev's work for the homogeneous gas. We believe that the approach presented in this paper is somewhat simpler to follow and more illustrative than previous methods. More importantly, it allows us to go beyond the weakly interacting limit in a very natural and straightforward extension of our treatment. It is this latter extension that will form the main part of this paper.

We will thus derive an expression for the condensate mean-field evolution in terms of the many-body transition (or simply $T$ ) matrix. The many-body approach we shall discuss is not limited to cases close to equilibrium, and therefore complements earlier work in this area. We would like to mention the work by Bijlsma and Stoof [19], who used a variational approach to calculate equilibrium properties, such as the normal and anomalous self-energies, in the many-body $T$-matrix approximation. The many-body $T$ matrix has also been recently discussed in the context of the homogeneous gas by Shi [20]. Furthermore, one of us has employed a functional formulation of the Keldysh theory to derive the time-dependent Landau-Ginzburg theory for the longwavelength dynamics of an inhomogeneous weakly interacting gas at nonzero temperatures, also in the many-body $T$-matrix approximation [21]. In this paper we will show, by different methods, how to derive a time-dependent general- 
ized nonlinear Schrödinger equation ${ }^{1}$ (NLSE) in a trap for both zero and nonzero temperatures based on microscopic arguments. We shall also discuss the physical significance of other contributions that are left out of such an equation, and thus discuss the validity of existing mean-field theories.

In an earlier publication [22], we derived equations of motion for thermal averages of products of up to three (single-particle) fluctuation operators. In this way, we obtained a time-dependent version $[23,24]$ of the Hartree-FockBogoliubov (HFB) equations [25-28] in terms of actual interatomic potentials, and further generalized them by considering more complex anomalous averages (triplets). This closed system of equations can be used in two different ways: in the first approach, all averages of products of fluctuation operators evolve on similar time scales, so that the equations need to be solved self-consistently. Such a treatment enables us to investigate the possibility of further order parameters being present in our system. We should note that the possibility of pairing as a competing transition to BEC in the case of attractive interactions has already been investigated in [29]. The equations of [22] further allow for the possibility of three atoms grouping together, such as, for example, condensation of triplets [30]. However, there are also situations (in particular a dilute gas with repulsive interactions), in which the higher-order (anomalous) correlations vary on faster time scales, enabling us to formally eliminate them from the equations of motion, i.e., by integrating over their effect during collisions. In this paper we will show how this formal (adiabatic) elimination of the pair correlation in our HFB equations gives rise to a nonlinear Schrödinger equation that includes the nondressed repeated binary interactions (ladders) at zero temperature. We shall furthermore extend our treatment to nonzero temperatures, for which we shall also need to eliminate the triplets mentioned above.

The equations of motion for averages of products of (up to three) single-particle fluctuation operators have been given in Appendix A, although we refer the reader to [22] for more details. We emphasize that these equations do not merely bring the bare particle ladder interactions into our formalism, but also include more complex effects, such as dressing and damping of intermediate states during collisions. In this paper, we discuss how - and in what limits - these microscopic equations reduce to the conventionally used phenomenological Gross-Pitaevskii expression. We also discuss the possibility of consistent theories outside these limits. Furthermore, we explicitly mention what processes must be neglected in order to obtain a NLSE. In fact, we shall see one has to neglect the effect of the condensate mean field and those due to the presence of excited atoms on the intermediate states of a binary collision [31]. Identification of these terms shows how to explore the deviation from the bareparticle $T$ matrix due to dressing generated by the mean fields. This treatment enables us to make qualitative predictions about the validity regime of the phenomenological GPE, an issue we hope to address computationally for inhomogeneous gases in the future.

\footnotetext{
${ }^{1}$ To avoid confusion, we shall henceforth refer to the conventionally used equation as the GPE and the one we shall be deriving here as the NLSE.
}

One might expect the zero-temperature evolution of the mean field of a trapped Bose-Einstein condensate in the simplest version of the mean-field theory, to be described - in the occupation number representation - by

$$
i \hbar \frac{d z_{n}(t)}{d t}=\sum_{k} h_{n k}^{(0)}(t) z_{k}(t)
$$

Here $z_{n}(t)$ corresponds to the time-dependent mean value amplitude of the $n$th trap level which is obtained from the single-particle operators $\hat{a}_{n}(t)$ according to the shift [28]

$$
\hat{a}_{n}(t)=z_{n}(t)+\hat{c}_{n}(t) .
$$

Here the operators $\hat{a}_{n}$ are defined by the usual decomposition of the Bose field operator $\hat{\Psi}(\mathbf{r}, t)$ into any complete set of orthonormal single-particle states $\psi_{n}(\mathbf{r})$, namely,

$$
\hat{\Psi}(\mathbf{r}, t)=\sum_{n} \psi_{n}(\mathbf{r}) \hat{a}_{n}(t)
$$

In this simplest approach, the time-dependent condensate mean field depends on the Hartree-Fock Hamiltonian $\mathbf{h}^{(\mathbf{0})}(t)$ given by

$$
h_{n k}^{(0)}(t)=\langle n|\hat{\Xi}| k\rangle+\sum_{i j}\langle n i|\hat{V}| j k\rangle z_{i}^{*}(t) z_{j}(t)
$$

Here $\hat{\Xi}$ contains the kinetic energy and trap potential and $\langle n i|\hat{V}| j k\rangle$ represents the symmetrized form of the actual (single-vertex) interatomic potential [32] between a pair of colliding particles. This is defined in terms of $\psi_{n}(\mathbf{r})$ by

$$
\langle n i|\hat{V}| j k\rangle=\frac{1}{2}\{(n i|\hat{V}| j k)+(n i|\hat{V}| k j)\}
$$

where

$$
(n i|\hat{V}| j k)=\iint d \mathbf{r} d \mathbf{r}^{\prime} \psi_{n}^{*}(\mathbf{r}) \psi_{i}^{*}\left(\mathbf{r}^{\prime}\right) V\left(\mathbf{r}-\mathbf{r}^{\prime}\right) \psi_{k}\left(\mathbf{r}^{\prime}\right) \psi_{j}(\mathbf{r})
$$

and $V\left(\mathbf{r}-\mathbf{r}^{\prime}\right)$ represents the actual interatomic potential experienced between two interacting atoms at each collisional vertex. Although Eq. (1) may on first sight appear to be equivalent to the conventional form of the GPE [4],

$$
\begin{aligned}
i \hbar \frac{\partial \Phi(\mathbf{r}, t)}{\partial t}= & \left(-\frac{\hbar^{2} \nabla_{\mathbf{r}}^{2}}{2 m}+V_{\text {trap }}(\mathbf{r})\right) \Phi(\mathbf{r}, t) \\
& +N U_{0}|\Phi(\mathbf{r}, t)|^{2} \Phi(\mathbf{r}, t)
\end{aligned}
$$

this is not the case. The GPE takes account of all (repeated) collisional processes via a pseudopotential of the form $V\left(\mathbf{r}-\mathbf{r}^{\prime}\right)=U_{0} \delta\left(\mathbf{r}-\mathbf{r}^{\prime}\right)$ where $U_{0}=4 \pi \hbar^{2} a / m$ [33]. On the contrary, the matrix element defined in Eq. (5) represents the instantaneous (i.e., single-vertex) interaction between two atoms as depicted in Fig. 1(a).

In this paper we will show that we can include the ladder diagrams of Fig. 1(b) into our formalism, by considering the evolution of correlations of products of fluctuation operators 


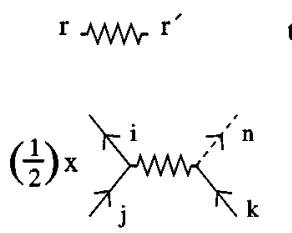
$\mathbf{t} \uparrow$

(a)

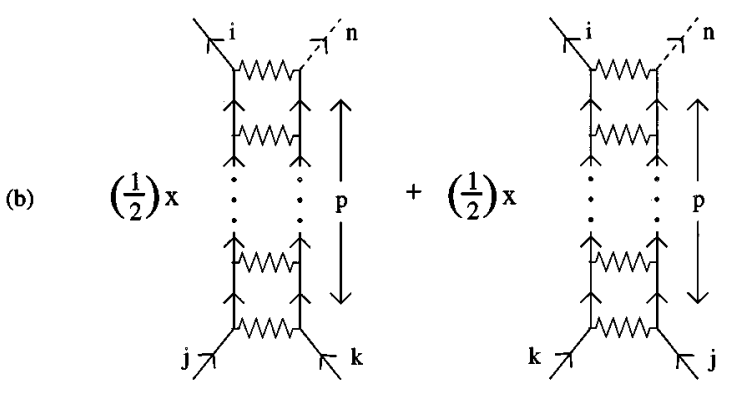

FIG. 1. (a) depicts the contribution to the evolution of the condensate mean field (level $n$ ) due to the instantaneous interaction of two condensate atoms (labeled by $j$ and $k$ ). In this, and all subsequent figures, the curly line represents the vertex between two interacting atoms located at $\mathbf{r}$ and $\mathbf{r}^{\prime}$, respectively, and time runs vertically upwards. We have explicitly drawn the two contributions to the evolution of the condensate mean field due to the physical nonsymmetrized matrix elements of Eq. (6) separately. Each of these diagrams is associated with a factor of $\left(\frac{1}{2}\right)$ into our final equation for the evolution of $z_{n}$, as defined by Eq. (5). In our notation, continuous lines with one "free" end point represent condensate particles, and have a factor $z$ associated with them (the dashed condensate line gives no such contribution, as it is the one whose evolution we are monitoring in this paper). (b) shows the cumulative effect of repeated binary interactions of this type, or ladder diagrams, in which an arbitrary number $p$ of loops $(p \geqslant 0)$ may be present. Here, the final scattering into (condensate) states $n$ and $i$ has been mediated by other excited states (given by the vertical lines with arrows).

$\hat{c}_{n}$ which are given in Appendix A. These correlations influence the condensate mean field according to the exact equation [22]

$$
\begin{aligned}
i \hbar \frac{d z_{n}}{d t}= & \sum_{k}\langle n|\hat{\Xi}| k\rangle z_{k}+\sum_{i j k}\langle n i|\hat{V}| j k\rangle\left[z_{i}^{*} z_{j} z_{k}+\kappa_{j k} z_{i}^{*}\right. \\
& \left.+2 \rho_{j i} z_{k}+\lambda_{i j k}\right]
\end{aligned}
$$

where we have defined the quantities

$$
\begin{gathered}
\rho_{j i}=\left\langle\hat{c}_{i}^{\dagger} \hat{c}_{j}\right\rangle, \quad \kappa_{j k}=\left\langle\hat{c}_{k} \hat{c}_{j}\right\rangle=\left\langle\hat{c}_{j} \hat{c}_{k}\right\rangle, \\
\lambda_{i j k}=\left\langle\hat{c}_{i}^{\dagger} \hat{c}_{j} \hat{c}_{k}\right\rangle=\left\langle\hat{c}_{k} \hat{c}_{j} \hat{c}_{i}^{\dagger}\right\rangle .
\end{gathered}
$$

In obtaining Eq. (8) we have merely assumed the existence of a mean field [in the sense of Eq. (2)] and that the dominant collisions in our atomic assembly occur pairwise.

The main part of this paper deals with the microscopic derivation of a nonlinear Schrödinger equation for bare particles in a trap, and we shall discuss in what limits it reduces to simpler versions. The use of a NLSE necessitates a welldefined and thus slowly varying condensate fraction, which in our formalism corresponds to a slowly evolving mean- field amplitude $z_{n}$, in comparison with the duration of a typical binary interaction. This means that, in most of the analysis given below, we are limiting ourselves to the case of net repulsive interactions between the atoms; i.e., we are not allowing for BCS-type effects [29] to become important. When extending our treatment to nonzero temperatures, we will similarly need to assume slow evolution of the excitedstate populations, although this issue becomes rather subtle as we shall discuss in Sec. III C. In our subsequent calculations we shall discuss when the thermal correlations $\kappa_{j k}$ and $\lambda_{i j k}$ vary on a much smaller time scale and show how they can be formally eliminated in our treatment.

This paper is divided into five parts. In the first part of the paper we restrict ourselves to near zero temperatures, for which it appears reasonable to neglect all effects of excited states. We thus obtain a NLSE that includes the full (bare particle) ladder interactions, where this effective interaction potential arises from the adiabatic elimination of the anomalous correlation $\kappa_{j k}$. This equation is then shown to reduce to the phenomenological Gross-Pitaevskii equation by means of the usual pseudopotential approximation. In Sec. III, we extend our treatment to nonzero temperatures. The first modification that comes about is the inclusion of the occupation of virtually excited states into the ladder diagrams. However, the finite number of excited atoms further necessitates the consideration of collisions between condensed and excited atoms. We thus show how adiabatic elimination of $\lambda_{i j k}$ "upgrades" the condensate-excited-state interaction potential to the ladder approximation. In Sec. IV, we show how the bare particle propagators get dressed by the condensate mean field and by interactions with other excited atoms and discuss the consequences of identifying these terms in our equations. We then discuss, in Sec. V, the validity of several mean-field theories for both zero and nonzero temperatures and in particular point out the inconsistency of the conventional finite-temperature mean-field approach (i.e., what is commonly referred to as HFB). Finally, in Sec. VI, we discuss some further effects of the mean fields on collisions. The detailed form of the equations needed for the current discussion has been reproduced in Appendix A, whereas Appendix B gives an exact treatment of the condensate mean-field evolution up to second order in the interaction potential.

\section{LADDER INTERACTIONS IN A BARE PARTICLE BASIS AT ZERO TEMPERATURES}

Let us initially assume that the atomic assembly is sufficiently dilute, so that we can treat the bare particles as weakly interacting quantities. In this limit, bare particles form a reasonable basis set for the system and can thus be used for the description of the assembly. We shall take the noninteracting part $\hat{\Xi}$ of the single bare-particle Hamiltonian in a trap as diagonal, by writing

$$
\langle n|\hat{\Xi}| k\rangle=\hbar \omega_{n} \delta_{k n}
$$

If this basis accurately represents the state of the system (i.e., all dressing of states by the mean fields can be ignored), then the anomalous average $\kappa$ will evolve rapidly, thus enabling us to adiabatically eliminate it. The elements of $\rho$, however, vary on a slower time scale, as their evolution depends on an 
energy difference. This means that they should, in principle, be retained in the equation of motion (8) for the condensate evolution. Near $T=0$, however, the occupation of excited states is extremely scarce, so that we can, in the first instance, neglect its effect on the condensate evolution.

Within these approximations, Eq. (8) reduces to

$$
i \hbar \frac{d z_{n}}{d t}=\hbar \omega_{n} z_{n}+\sum_{i j k}\langle n i|\hat{V}| j k\rangle\left[z_{i}^{*} z_{j} z_{k}+\kappa_{j k} z_{i}^{*}\right] .
$$

In order to adiabatically eliminate the pairing $\kappa_{j k}$, we shall need its equation of motion given in Appendix A. We note that in a bare particle basis at $T=0$, Eq. (A6) essentially simplifies to

$$
i \hbar \frac{d \kappa_{k j}}{d t}=\hbar\left(\omega_{k}+\omega_{j}\right) \kappa_{k j}+\Delta_{k j}
$$

where we have defined [28]

$$
\Delta_{k j}=\sum_{m s}\langle k j|\hat{V}| m s\rangle\left[z_{m} z_{s}+\kappa_{m s}\right]
$$

We are now in a position to derive a NLSE valid at $T$ $=0$ with the actual interatomic potential of Eq. (4) replaced by the two-body $T$ matrix. Such an equation will be shown to be equivalent to the conventional GPE if we replace the $T$ matrix by the zero-range pseudopotential. This procedure is analogous to that pursued by Beliaev [15] for the homogeneous gas. The advantage of the approach presented here is that it enables us to give criteria for the validity of the conventional approach and its application to the case of trapped gases.

Integrating Eq. (12) and substituting into Eq. (11), we obtain

$$
\begin{aligned}
i \hbar \frac{d z_{n}}{d t}= & \hbar \omega_{n} z_{n}+\sum_{i m s}\langle n i|\hat{V}| m s\rangle z_{i}^{*} z_{m} z_{s}+\sum_{i j k}\langle n i|\hat{V}| j k\rangle z_{i}^{*}(t) \\
& \times \int \frac{d t^{\prime}}{i \hbar} e^{-i\left(\omega_{k}+\omega_{j}\right)\left(t-t^{\prime}\right)} \sum_{m s}\langle j k|\hat{V}| m s\rangle\left[z_{m}\left(t^{\prime}\right) z_{s}\left(t^{\prime}\right)+\kappa_{m s}\left(t^{\prime}\right)\right],
\end{aligned}
$$

where the integration is to be carried out in the interval $\left[t_{0}, t\right]$, in which the collision takes place. Since the gas is considered sufficiently dilute, the mean collisional duration is negligible compared to the time between successive collisions, enabling us to take the limit $t_{0} \rightarrow-\infty$. In writing Eq. (14) we have made use of the approximation that the pairing $\kappa$ asymptotically relaxes to zero, so that $\kappa_{j k}\left(t_{0}\right) \rightarrow 0$ as $t_{0}$ $\rightarrow-\infty$. Physically, this is the same as saying that these anomalous interparticle correlations are dominantly created within a collision between condensate particles.

A further crucial point to note is that, since we are trying to derive a NLSE, we are implicitly assuming that the mean value amplitude $z_{i}(t)$ is a slowly varying quantity, that is, it remains practically constant over the time scale in which the correlation $\kappa_{j k}$ varies. Thus, we are justified in allowing the quantities $z_{i}\left(t^{\prime}\right)$ present in the integrand of Eq. (14) to evolve freely with time by setting

$$
z_{i}\left(t^{\prime}\right)=z_{i}(t) e^{+i \omega_{i}\left(t-t^{\prime}\right)}
$$

If we now intitially limit ourselves to second order in the interaction potential $V$, we note that we can disregard the $\kappa_{m s}\left(t^{\prime}\right)$ term of Eq. (14), as its first contribution will be to order $V^{3}$. Then, Eq. (14) reduces to

$$
i \hbar \frac{d z_{n}}{d t}=\hbar \omega_{n} z_{n}+\sum_{i m s}\left\langle n i\left|\hat{T}^{2 \mathrm{~B}}(E)\right| m s\right\rangle z_{i}^{*} z_{m} z_{s},
$$

which gives us the bare-particle two-body $T$ matrix in a trap to second order in the interaction potential $\hat{V}$, namely,

$$
\hat{T}^{2 \mathrm{~B}}(E)=\hat{V}+\sum_{j k} \hat{V}|j k\rangle \int \frac{d t^{\prime}}{i \hbar} e^{-i\left(\omega_{k}+\omega_{j}-E\right)\left(t-t^{\prime}\right)}\langle j k| \hat{V} .
$$

Here $E$ represents the energy of the colliding (bare) atoms prior to the collision in the center-of-mass frame [i.e., in Eq. (16), $\left.E=\hbar\left(\omega_{m}+\omega_{s}\right)\right]$, whereas $j$ and $k$ correspond to intermediate trap eigenstates induced during the collision. However, we point out that it is precisely the presence of the term $\kappa_{m s}\left(t^{\prime}\right)$, which generates $T^{2 \mathrm{~B}}$ to all orders in $V$, as can easily be seen by repeated use of Eqs. (11)-(13). We have hence shown Eq. (16) to be valid to all orders in $V$, with $\hat{T}^{2 \mathrm{~B}}$ satisfying the following Lippmann-Schwinger relation [34] in a trap

$$
\begin{aligned}
\hat{T}^{2 \mathrm{~B}}(E)= & \hat{V}+\sum_{j k} \hat{V}|j k\rangle \int \frac{d t^{\prime}}{i \hbar} e^{-i\left(\omega_{k}+\omega_{j}-E\right)\left(t-t^{\prime}\right)} \\
& \times\langle j k| \hat{T}^{2 \mathrm{~B}}(E) .
\end{aligned}
$$

This modified form of the NLSE accurately represents a collision between two condensate atoms occurring in a trap in the absence of other particles, by taking account of all possible states via which the collision can proceed. Thus, Eq. (18) includes the ladder diagrams for bare particles as depicted diagrammatically in Fig. 2(a).

In the above derivation of Eq. (16), we have repeatedly used the assumption that the mean value of the condensate field evolves more slowly than the anomalous average $\kappa$ we have adiabatically eliminated. This assumption is indeed valid for the weakly interacting gas, as explained below. In the low-temperature case $(k T \ll \hbar \omega)$ discussed in this sec- 
(a)

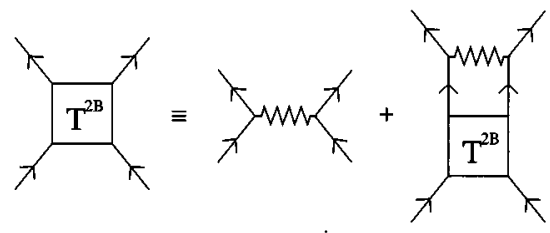

(b)

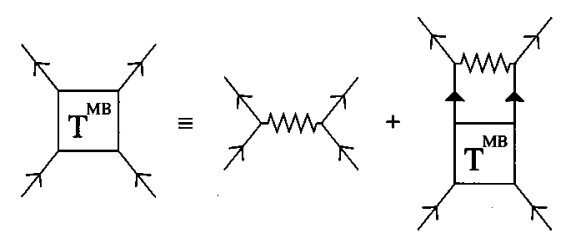

(c)

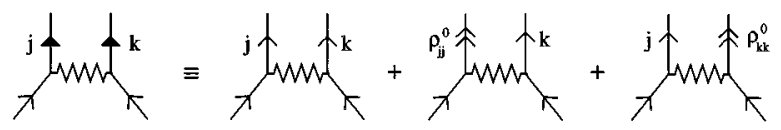

FIG. 2. (a) depicts diagrammatically the integral definition of the bare-particle two-body $T$ matrix, as defined by Eq. (18). In (b) and (c) we have illustrated the definition of the many-body $T$ matrix according to Eq. (26). The new feature introduced in (b) is that one now takes account of the occupation of the intermediate states through which the collision proceeds. Thus, the thick arrows indicate that the collisions actually occur in an atomic medium, as opposed to the vacuum. In particular, (c) indicates explicitly how this excited-state occupation is taken into account according to the many-body factor $\left(1+\rho_{j j}^{0}+\rho_{k k}^{0}\right)$, with the $\rho^{0}$ term indicated by the double arrow.

tion, it suffices to show that the amplitude of states transiently populated in a two-body collision varies very rapidly, i.e., we can neglect any modifications on the two-body ladder diagrams of Fig. 1(b). The amplitude of these transient excited states evolves with a typical frequency $\hbar / m a^{2}$. This should be compared with a typical frequency for the evolution of the condensate mean field, e.g., the frequency of collective modes that are excited in experiments. In the case of a trapped condensate, this is given approximately by the trap frequency $\hbar / m l_{0}^{2}$, where $l_{0}$ corresponds to the width of the ground state in the confining potential. In all cases, $l_{0} \gg a$, with typical values of the order of $100 a$, confirming the va- lidity of our earlier assumption that the mean values evolve more slowly than the other processes occurring in the middle of a collision.

In upgrading Eq. (16), the natural next step would be to introduce the many-body $T$ matrix, which takes account of the occupation of such transient excited states; this could be done by making use of the next set of terms in $d \kappa / d t$ [given in Eq. (A6)]. However, before returning to this in Sec. III A, let us focus on the relation between this version of the microscopically derived zero-temperature NLSE (16) and the phenomenological GPE (7).

\section{A. Reduction to the Gross-Pitaevskii equation}

Let us now show explicitly how we can obtain the GPE from our NLSE (16). To do this we shall need to approximate the (bare-particle) $T$-matrix defined in Eq. (18) in terms of actual interatomic potentials, by a phenomenological model, such as the pseudopotential approach discussed in detail in the classic paper by Huang and Yang [17]. For the low condensation temperatures involved, we can limit ourselves to the lowest-order contribution of the $s$-wave. We may thus write

$$
\hat{T}^{2 B}(\mathbf{r}) \simeq \frac{4 \pi \hbar^{2} a}{m} \delta(\mathbf{r}),
$$

where $a$ corresponds to the $s$-wave scattering length.

Let us now use this approximation in Eq. (16) to explicitly reconstruct Eq. (7). In fact, in order to reconstruct the GPE, we have to explicitly express the diagonal element $\hbar \omega_{n} z_{n}$ of Eq. (16) as $\Sigma_{k}\langle n|\hat{\Xi}| k\rangle z_{k}$. We shall now also multiply the resulting equation by $\phi_{n}(\mathbf{r}) / \sqrt{N}$ and sum over the indices $n$ [so as to obtain the desired $\Phi(\mathbf{r}, t)$ on the left-hand side of our equation]. We note that the factor of $\sqrt{N}$ is needed so as to renormalize the wave functions, as the $z_{i}$ satisfy

$$
\sum_{i} z_{i}^{*} z_{i}=N
$$

After carrying out the integration with respect to the $\delta$ function and rearranging, we thus obtain

$$
\begin{aligned}
i \hbar \frac{\partial}{\partial t} \Phi(\mathbf{r}, t)= & \int d \mathbf{r}^{\prime}\left[\sum_{n} \phi_{n}^{*}\left(\mathbf{r}^{\prime}\right) \phi_{n}(\mathbf{r})\right] \hat{\Xi}\left(\mathbf{r}^{\prime}\right) \sum_{k} \phi_{k}\left(\mathbf{r}^{\prime}\right) \frac{z_{k}(t)}{\sqrt{N}} \\
& +N U_{0} \int d \mathbf{r}^{\prime}\left[\sum_{n} \phi_{n}^{*}\left(\mathbf{r}^{\prime}\right) \phi_{n}(\mathbf{r})\right] \sum_{i} \phi_{i}^{*}\left(\mathbf{r}^{\prime}\right) \frac{z_{i}^{*}(t)}{\sqrt{N}} \sum_{j} \phi_{j}\left(\mathbf{r}^{\prime}\right) \frac{z_{j}(t)}{\sqrt{N}} \sum_{k} \phi_{k}\left(\mathbf{r}^{\prime}\right) \frac{z_{k}(t)}{\sqrt{N}} .
\end{aligned}
$$

Using the completeness relation for orthogonal states, this reduces to the GPE (7) for $\Phi(\mathbf{r}, t)$.

One of the aims of this paper is to specify validity regimes for the currently existing mean-field theories, including the GPE. However, some limitations of the GPE have been already extensively discussed in the literature. We shall briefly discuss those here, so as to provide a complete account of potential shortcomings of the GPE.

Firstly, we should emphasize that the pseudopotential should not be used as a straightforward substitute for the actual interatomic potential. To explain what we mean by this statement, we shall now outline the simplest possible 
derivation of the GPE, following [35]. We start with the Heisenberg equation of motion for the Bose field operator $\hat{\Psi}(\mathbf{r}, t)$ in the limit of pairwise interactions. After using the appropriate commutation relations for the field operators and taking mean values, we find

$$
\begin{aligned}
i \hbar \frac{\partial \Phi(\mathbf{r}, t)}{\partial t}= & \left(-\frac{\hbar^{2} \nabla_{\mathbf{r}}^{2}}{2 m}+V_{\text {trap }}(\mathbf{r})\right) \Phi(\mathbf{r}, t)+\int d \mathbf{r}^{\prime} V\left(\mathbf{r}-\mathbf{r}^{\prime}\right) \\
& \times\left\langle\hat{\Psi}^{\dagger}\left(\mathbf{r}^{\prime}, t\right) \hat{\Psi}\left(\mathbf{r}^{\prime}, t\right) \hat{\Psi}(\mathbf{r}, t)\right\rangle,
\end{aligned}
$$

where $V\left(\mathbf{r}-\mathbf{r}^{\prime}\right)$ again corresponds to the actual potential experienced by a pair of interacting atoms (assuming a central potential).

In the simplest derivations, one first uses the pseudopotential as a model interaction via $V\left(\mathbf{r}-\mathbf{r}^{\prime}\right)=U_{0} \delta\left(\mathbf{r}-\mathbf{r}^{\prime}\right)$ and then drops the interatomic correlations by setting $\left\langle\hat{\Psi}^{\dagger}\left(\mathbf{r}^{\prime}, t\right) \hat{\Psi}\left(\mathbf{r}^{\prime}, t\right) \hat{\Psi}(\mathbf{r}, t)\right\rangle=\left\langle\hat{\Psi}^{\dagger}\left(\mathbf{r}^{\prime}, t\right)\right\rangle\left\langle\hat{\Psi}\left(\mathbf{r}^{\prime}, t\right)\right\rangle\langle\hat{\Psi}(\mathbf{r}, t)\rangle$. The two steps are, of course, interlinked, with the second step justified by arguing that the effect of all correlations thus neglected is taken into account in the effective interaction strength $U_{0}$.

However, we wish to stress that an effective interaction treatment can only be rigorously justified if the pseudopotential approximation is carried out after the two-body interaction potential has been "upgraded" to the $T$ matrix, in the manner shown above. This is not a new result: in fact, the replacement of the interaction potential $V$ by the two-body $T$ matrix has been extensively discussed in the literature. The most notable work is the diagrammatic approach discussed for the homogeneous gas by Beliaev [15], and Popov's definition of a suitable effective action for the dilute Bose gas [16]. Nonetheless, we feel that this point is often overlooked in current treatments of the Bose gas, and we have therefore chosen to stress it in the first part of this paper. In fact, it is only possible to apply the pseudopotential approximation to $V$ (and drop all interatomic correlations as discussed earlier) in the limit of weak interactions, as defined by [14]. In general, one must go beyond this limit, which mathematically corresponds to defining an effective interaction beyond the two-body $T$-matrix approximation. Such an improved effective interaction can no longer be represented by the usual $s$-wave scattering length pseudopotential, and will, thus, yield an equation distinct from the GPE, as will be discussed in subsequent sections. In essence, this means that the replacement of $V$ by the pseudopotential has a limited range of validity due to the effect of the medium in which the collisions occur, as will be explained shortly.

However, let us for the moment discuss other limitations of the GPE that are inherent in the nature of the pseudopotential approximation (19) (and will thus still be important even when this is correctly applied on the two-body $T$ matrix). For example, it is well known in the case of the homogeneous gas, that the use of a $\delta$-function potential in a selfconsistent treatment can lead to unphysical results, such as no depletion of the condensate [36]. This can be easily understood by looking at the original work by Huang and Yang [17]. The reason for such unphysical results is that the pseudopotential approximation already contains an implicit assumption about the actual interaction operator, namely, that it is acting on free particle states [37]. In terms of Popov's approach [16], this means that a $\delta$-function potential can only be used in combination with an ultraviolet cutoff in the theory. Furthermore, the nature of the pseudopotential approximation explicitly ignores all momentum dependence, which appears in the problem as higher-order terms in the $s$-wave scattering length $a$. This is an excellent approximation, valid, in the case of the homogeneous gas if $a / \Lambda \ll 1$, where $\Lambda$ represents the atomic de Broglie wavelength. It cannot, therefore, be put into into higher-order terms in a perturbation expansion with an unrestricted summation over momenta. Thus far, in this section, we have focused our attention on the limitations of the GPE in terms of the treatment of the spatial and momentum dependence of the atomic interactions. These limitations are well known and have been previously dealt with in the literature. The treatment presented in this paper is one way of overcoming such difficulties and handling spatially dependent interaction effects that are not present in the phenomenological Gross-Pitaevskii model [38]. However, these limitations are not the only problem one has to address. In fact, the main emphasis of this paper lies on the extension of the effective interaction approach beyond the weakly interacting (GPE) limit, and it is to this issue that we now turn our attention. We remind the reader that the derivation of Eq. (16) found in the preceding section is based on a bare particle basis. This implies, for example, that we have ignored all dressing effects on the intermediate collisional states due to the condensate mean field. This obviously means that such effects are also implicitly ignored in the GPE, and implies that the GPE is strictly only valid if the atoms are colliding in a strictly binary manner. The reason these mean field effects cannot be included in a phenomenological GPE based on the s-wave scattering length $a$, is that, in the experiments, $a$ is spectroscopically determined in the absence of mean fields (i.e., effectively in vacuum). Our microscopic approach fully treats collisions in the presence of mean fields, and we believe it provides a natural formalism for obtaining expressions for both dressing and damping of the GPE [39].

\section{LADDER DIAGRAMS AT FINITE TEMPERATURES IN A BARE PARTICLE BASIS}

Following the analysis of the previous section, it should be clear to the reader that the GPE represents only a limiting case of the equations given in Appendix A, since it neglects the effect of the medium in which the collisions occur (which becomes increasingly important as the atomic assembly becomes denser). Before we deal with these issues, we would, however, first like to extend Eq. (16) to finite temperatures, which corresponds to a well-posed problem within our formalism.

When extending our treatment to nonzero temperatures, a variety of new features arises: firstly, there will now be a non-negligible occupation of excited states, which leads to a modification in the scattering between two condensed atoms, as discussed in Sec. III A below. At the same time, however, the evolution of the condensate mean field will also be affected by collisions between condensed and excited atoms, as will be dealt with in Sec. III B.

In this section we discuss the limit of weak interactions, 
in which the collisions can be described in terms of bare (i.e., non-dressed) atoms. The deviation from such a simplistic picture (due to dressing induced by mean fields) will be dealt with in Sec. IV.

\section{A. The many-body $T$ matrix for weakly interacting particles}

We shall now deal with the first of the finite temperature effects, namely, how Bose statistics affect the $T$ matrix, by taking account of the occupation of low-lying excitations during a collision between two condensate particles. This effect is expected to be so small at low temperatures, that we have chosen to neglect it altogether near $T=0$. However, in order to take account of the occupation of virtual states accessed during a collision between two condensate particles, we need to modify Eq. (12) to

$$
i \hbar \frac{d \kappa_{k j}}{d t}=\hbar\left(\omega_{k}+\omega_{j}\right) \kappa_{k j}+\Delta_{k j}+\sum_{r}\left[\rho_{k r} \Delta_{r j}+\Delta_{k r} \rho_{r j}^{*}\right]
$$

We again formally integrate the above equation and adiabatically eliminate $\kappa_{j k}$ from Eq. (11). Doing so we obtain, to second order in the interaction potential, the following equation of motion for the condensate:

$$
\begin{aligned}
i \hbar \frac{d z_{n}}{d t}= & \hbar \omega_{n} z_{n}+\sum_{i m s}\langle n i|\hat{V}| m s\rangle z_{i}^{*} z_{m} z_{s}+\sum_{i j k}\langle n i|\hat{V}| j k\rangle z_{i}^{*}(t) \int \frac{d t^{\prime}}{i \hbar} e^{-i\left(\omega_{k}+\omega_{j}\right)\left(t-t^{\prime}\right)} \sum_{m s}\langle j k|\hat{V}| m s\rangle z_{m}\left(t^{\prime}\right) z_{s}\left(t^{\prime}\right) \\
& +\sum_{i j k}\langle n i|\hat{V}| j k\rangle z_{i}^{*}(t)\left\{\int \frac{d t^{\prime}}{i \hbar} \sum_{r m s} e^{-i\left(\omega_{k}+\omega_{j}\right)\left(t-t^{\prime}\right)}\langle r k|\hat{V}| m s\rangle z_{m}\left(t^{\prime}\right) z_{s}\left(t^{\prime}\right) \rho_{j r}\left(t^{\prime}\right)+\{k \leftrightarrow j\}\right\} .
\end{aligned}
$$

Here, the notation $+\{k \leftrightarrow j\}$ indicates the presence of an identical term upon interchanging the (intermediate propagation) labels $j$ and $k$, and thus indicates the presence of both a direct and an exchange term [in much the same manner as those are included in the symmetrization of the interaction vertex (5)]. Once again the $\kappa$ correlations ensure that this equation generalizes to all $V$. Hence, $T^{2 \mathrm{~B}}$ in Eq. (16) becomes replaced by the operator $\hat{t}$, defined by

$$
\begin{aligned}
\hat{t}(E)= & \hat{V}+\sum_{j k} \hat{V}|j k\rangle \int \frac{d t^{\prime}}{i \hbar} e^{-i\left(\omega_{k}+\omega_{j}-E\right)\left(t-t^{\prime}\right)}\left\langle j k\left|\hat{t}(E)+\sum_{r j k} \hat{V}\right| j k\right\rangle \\
& \times\left\{\int \frac{d t^{\prime}}{i \hbar} e^{-i\left(\omega_{k}+\omega_{j}-E\right)\left(t-t^{\prime}\right)} \rho_{j r}\left(t^{\prime}\right)\langle r k|+(k \leftrightarrow j)\right\} \hat{t}(E) .
\end{aligned}
$$

In the limit of weak interactions, we can treat the elements of $\rho$ as diagonal, thus corresponding to population of excited states. The off-diagonal elements will correspond to dressing of the bare atoms and will be discussed in Sec. IV. In this manner, we arrive at the Lippmann-Schwinger relation for the many-body $T$ matrix in a trap in terms of bare particles, in the form

$$
\hat{T}^{\mathrm{MB}}(E)=\hat{V}+\sum_{j k} \hat{V}|j k\rangle \int \frac{d t^{\prime}}{i \hbar} e^{-i\left(\omega_{k}+\omega_{j}-E\right)\left(t-t^{\prime}\right)}\left[1+\rho_{j j}^{0}\left(t^{\prime}\right)+\rho_{k k}^{0}\left(t^{\prime}\right)\right]\langle j k| \hat{T}^{\mathrm{MB}}(E) .
$$

This definition has also been depicted diagramatically in Figs. 2(b)-2(c). The operator $\hat{T}^{\mathrm{MB}}$ of Eq. (26) is linked to $\hat{T}^{2 \mathrm{~B}}$ of Eq. (18) by the equivalent definition

$$
\hat{T}^{\mathrm{MB}}(E)=\hat{T}^{2 \mathrm{~B}}(E)+\sum_{j k} \hat{T}^{2 \mathrm{~B}}(E)|j k\rangle \int \frac{d t^{\prime}}{i \hbar} e^{-i\left(\omega_{k}+\omega_{j}-E\right)\left(t-t^{\prime}\right)}\left[\rho_{j j}^{0}\left(t^{\prime}\right)+\rho_{k k}^{0}\left(t^{\prime}\right)\right]\langle j k| \hat{T}^{\mathrm{MB}}(E) .
$$

It is easy to see that, in the homogeneous limit, the expression (26) reduces to the well-known integral relation for the many-body $T$ matrix [40], namely,

$$
T^{\mathrm{MB}}\left(k^{\prime}, k, K ; E\right)=V\left(k^{\prime}-k\right)+\int \frac{d k^{\prime \prime}}{(2 \pi)^{3}} V\left(k^{\prime}-k^{\prime \prime}\right) \frac{1+N\left(K / 2+k^{\prime \prime}\right)+N\left(K / 2-k^{\prime \prime}\right)}{E-\left(\hbar k^{\prime \prime}\right)^{2} / m+i 0} T^{\mathrm{MB}}\left(k^{\prime \prime}, k, K ; E\right) .
$$


Here $k$ and $k^{\prime}$ respectively correspond to ingoing and outcoming particle momenta, $K$ to the center-of-mass momentum, and $E$ represents the kinetic energy for the two atoms in the center-of-mass frame. Furthermore, $N$ represents the average occupation number of a single-particle state (of specified momentum).

In Sec. III, we have already explained that the lowtemperature collisional evolution of the condensate mean field in the weakly interacting limit is governed by the twobody $T$ matrix. Justifying why $\hat{T}^{\mathrm{MB}}$ is the suitable parameter in the regime $k T \gg \hbar \omega$ is more tricky. One of us has shown elsewhere [21] that the relevant time scale for the homogeneous gas is given by $\hbar / k T$. This can be interpreted as the time scale for atoms to move a typical interparticle separation, a sensible time for the manifestation of many-body effects. These effects give rise to a slower evolution in comparison with the two-body one. Nonetheless, the time scale for the many-body $T$ matrix is still much faster than the collective motion in a trap $\left(\hbar / m l_{0}^{2}\right)$, thus justifying once again the adiabatic elimination of the anomalous average and the free evolution of the condensate mean field (16).

\section{B. Ladder approximation to the condensate-excited-state interactions}

So far, we have only accounted for the occupation of states through which a condensate-condensate collision proceeds, in terms of the many-body $T$ matrix. The other important effect arising at finite temperatures is the interaction between condensed and excited atoms. Obviously, such an effect must also be included in a NLSE describing the evolution of the condensate. To deal with this effect, we must now also consider the rest of Eq. (8) which has been ignored up to this point. To be more precise, we need to consider the effect of the additional contribution

$$
i \hbar \frac{d z_{n}}{d t}=\cdots+\sum_{i j k}\langle n i|\hat{V}| j k\rangle\left[2 \rho_{j i} z_{k}+\lambda_{i j k}\right] .
$$

Careful observation of the first few terms in the equation of motion for $\lambda_{i j k}$, Eq. (A7) suggests, by analogy to the arguments of Sec. II, that it is indeed the suitable quantity for upgrading the actual (single-vertex) interatomic potential for $2 \rho_{j i} z_{k}$ in Eq. (29) to the $T$-matrix level. However, we note that the triplet $\lambda$ is not included (i.e., $\lambda=0$ ) in the traditional mean field (i.e., HFB) theory. This shows that we need to go beyond HFB in order to rigorously obtain a NLSE for the evolution of the condensate mean field at non-zero temperatures, and we shall discuss this below. An extension beyond HFB is, strictly speaking, also essential for a consistent $T$ $=0$ theory.

We shall now also adiabatically eliminate [41] the quantity $\lambda_{i j k}$ appearing in Eq. (29). Consider initially the contribution

$$
\begin{aligned}
i \hbar \frac{d}{d t}\left(\lambda_{i j k}\right)= & \hbar\left(\omega_{k}+\omega_{j}-\omega_{i}\right) \lambda_{i j k} \\
& +\sum_{m s}\langle j k|\hat{V}| m s\rangle\left(2 \rho_{m i} z_{s}+\lambda_{i m s}\right) .
\end{aligned}
$$

Thus, Eq. (29) becomes, to second order in the interaction potential

$$
\begin{aligned}
i \hbar \frac{d z_{n}}{d t}= & \cdots+\sum_{i m s}\langle n i|\hat{V}| m s\rangle 2 \rho_{m i} z_{s} \\
& +\sum_{i j k}\langle n i|\hat{V}| j k\rangle \int \frac{d t^{\prime}}{i \hbar} e^{-i\left(\omega_{k}+\omega_{j}-\omega_{i}\right)\left(t-t^{\prime}\right)} \\
& \times \sum_{m s}\langle j k|\hat{V}| m s\rangle 2 \rho_{m i}\left(t^{\prime}\right) z_{s}\left(t^{\prime}\right) .
\end{aligned}
$$

As before, we assume that all mean value amplitudes $z_{i}(t)$ vary on a much slower time scale than the anomalous correlations. Furthermore, we limit our discussion here to the slowly evolving diagonal elements $\rho_{i i}^{0} \delta_{m i}$ in a bare particle basis, for which $\rho_{i i}^{0}\left(t^{\prime}\right)=\rho_{i i}^{0}(t)$. This simplification avoids potential problems associated with the variation of $\rho$ being slow in comparison with the two-body $T$ matrix. We thus obtain the desired upgrading of condensate-excited-state interactions to the two-body $T$ matrix, valid to second order in $V$, just like in Eq. (17). Once again, we see that this expression can be generalized to all orders in the interaction potential, by taking account of the last term proportional to $\lambda_{i m s}$ in the right-hand side of Eq. (30).

Thus far, we have shown how to treat the condensatecondensate interactions to the bare-particle $\hat{T}^{\mathrm{MB}}$ level and the condensate-excited-state contributions to $\hat{T}^{2 \mathrm{~B}}$, so that the condensate mean field evolves according to the equation

$$
\begin{aligned}
i \hbar \frac{d z_{n}}{d t}= & \hbar \omega_{n} z_{n}+\sum_{i m s}\left\langle n i\left|\hat{T}^{\mathrm{MB}}\right| m s\right\rangle z_{i}^{*} z_{m} z_{s} \\
& +2 \sum_{i s}\left\langle n i\left|\hat{T}^{2 B}\right| i s\right\rangle \rho_{i i}^{0} z_{s} .
\end{aligned}
$$

It is clear that a consistent finite-temperature theory would require the latter contribution to be also expressed in terms of $\hat{T}^{\mathrm{MB}}$. The subtle point that needs to be addressed here is that such an expression will contain terms of order $\left(\rho^{0}\right)^{2}$; hence, we expect (and we shall indeed confirm in our detailed treatment below) that in this situation, we should also take account of the scattering of excited states into the condensate, which modifies the evolution of the condensate mean value.

\section{Interactions between excited states}

In this section we shall discuss how to upgrade expression (32) to the many-body $T$ matrix for excited states. On first inspection, this appears to be quite straightforward, upon considering the first three terms of Eq. (A7), namely, the contribution of 


$$
i \hbar \frac{d}{d t}\left(\lambda_{i j k}\right)=\hbar\left(\omega_{j}+\omega_{k}-\omega_{i}\right) \lambda_{i j k}+\sum_{m s}\langle j k|\hat{V}| m s\rangle\left(2 \rho_{m i} z_{s}+\lambda_{i m s}\right)+\left\{\sum_{r m s}\langle r k|\hat{V}| m s\rangle \rho_{j r}\left[2 \rho_{m i} z_{s}+\lambda_{i m s}\right]+(k \leftrightarrow j)\right\}
$$

to the equation for condensate evolution (29).

However, we wish to point out that maintaining merely this contribution for $\lambda_{i j k}$ cannot be consistent. The reason for this is that the next term in Eq. (A7), namely,

$$
i \hbar \frac{d}{d t}\left(\lambda_{i j k}\right)=\cdots-\left\{\sum_{r m s}\langle m s|\hat{V}| i r\rangle \rho_{j m} \rho_{k s} z_{r}+(k \leftrightarrow j)\right\}
$$

is an equally valid contribution to order $V^{2}$ in the expression for $d z_{n} / d t$. Thus, a consistent approach must simultaneously take into account both contributions (33) and (34) in adiabatically eliminating $\lambda$. The interpretation of the contribution (33) is straightforward: it leads to the renormalization of the condensate-excited-state interaction to $\hat{T}^{\mathrm{MB}}$. At first sight, it appears hard to interpret the physical significance of Eq. (34). To achieve this, we shall once again limit ourselves to the slowly evolving excited-state contributions $\rho^{0}$. In this limit, maintaining both terms (33) and (34), we obtain for the contribution of excited states to the evolution of the mean value amplitude $z_{n}$ to second order in $V$,

$$
\begin{aligned}
i \hbar \frac{d z_{n}}{d t}= & \cdots+2 \sum_{i s}\langle n i|\hat{V}| i s\rangle \rho_{i i}^{0} z_{s}+2 \sum_{i j k s}\langle n i|\hat{V}| j k\rangle \int \frac{d t^{\prime}}{i \hbar} e^{-i\left(\omega_{k}+\omega_{j}-\omega_{i}-\omega_{s}\right)\left(t-t^{\prime}\right)} \\
& \times\left[\rho_{i i}^{0}+\rho_{i i}^{0} \rho_{j j}^{0}+\rho_{i i}^{0} \rho_{k k}^{0}-\rho_{j j}^{0} \rho_{k k}^{0}\right]\left(t^{\prime}\right)\langle j k|\hat{V}| i s\rangle z_{s}(t)
\end{aligned}
$$

We can now easily identify the term in square brackets as

$$
\left(\rho_{j j}^{0}+1\right)\left(\rho_{k k}^{0}+1\right) \rho_{i i}^{0}-\rho_{j j}^{0} \rho_{k k}^{0}\left(\rho_{i i}^{0}+1\right) .
$$

This contribution is known to give the correct amplitude for the scattering of quasiparticles, and can be used to predict condensate lifetimes. This expression is also in agreement with the results of the functional integral approach developed independently by one of us $[21,42]$.

Hence, we can now identify the negative contribution in Eq. (34) as the term that ensures the correct factors for scattering into the condensate, due to the interaction of an excited state either with a condensed, or with another excited atom. Correct treatment of the factors of Eq. (36) to all orders in the interaction potential should lead to an additional term in the finite temperature NLSE, which should thus be expressible as

$$
\begin{aligned}
i \hbar \frac{d z_{n}}{d t}= & \hbar \omega_{n} z_{n}+\sum_{i m s}\left\langle n i\left|\hat{T}^{\mathrm{MB}}\right| m s\right\rangle z_{i}^{*} z_{m} z_{s}+2 \sum_{i s}\left\langle n i\left|\hat{T}^{\mathrm{MB}}\right| i s\right\rangle \rho_{i i}^{0} z_{s} \\
& -2 \sum_{i j k s}\left\langle n i\left|\hat{T}^{\mathrm{MB}}\right| j k\right\rangle \int \frac{d t^{\prime}}{i \hbar} e^{-i\left(\omega_{j}+\omega_{k}-\omega_{i}-\omega_{s}\right)\left(t-t^{\prime}\right)}\left[\rho_{j j}^{0} \rho_{k k}^{0}\right]\left(t^{\prime}\right)\left\langle j k\left|\hat{T}^{\mathrm{MB}}\right| i s\right\rangle z_{s} .
\end{aligned}
$$

Equation (37) is depicted diagrammatically in Fig. 3. This equation, already derived using an alternative approach in [21], is very important, as it includes the effect of kinetics [43] due to interactions of two excited atoms.

We note that a systematic analysis of the contributions (33)-(34) to all orders in $V$ appears to generate only one of the many-body $T$ matrices appearing in the latter term of Eq. (37). In particular, we only obtain $\langle j k|\hat{V}| i s\rangle$, instead of the anticipated $\left\langle j k\left|\hat{T}^{\mathrm{MB}}\right| i s\right\rangle$, which may at first glance appear unsatisfactory.

Let us, however, reexamine the derivation [22] of the equations of motion appearing in Appendix A. In their derivation, we have only maintained correlations of products of up to three fluctuation operators; in particular, we have treated the correlations of products of four such operators in their mean-field contributions, i.e., in terms of products of averages of two operators [or Wick's theorem, see Eq. (22) of Ref. [22]]. Since such correlations appear explicitly in the equation of motion for the normal average $\rho$, it is reasonable that the evolution of $\rho$, and therefore the collisions between two excited states, will only be treated in an exact manner, when the equation of motion for $\left\langle c^{\dagger} c^{\dagger} c c\right\rangle$ is explicitly worked out (for which the decomposition approximation is only carried out on higher-order correlations). This argument is justified by the fact that suitable treatment of $\left\langle c^{\dagger} c^{\dagger} c c\right\rangle$ gives rise, in the limit of no condensation [44], to the wellknown quantum Boltzmann equation [39]. Thus a full treatment of these more complex correlations can solve the apparent limitation of our formalism in terms of the derivation of Eq. (37), as discussed in Proukakis [39].

We would like to point out, however, that since the last (kinetic) contribution arises due to the interaction of two excited atoms resulting in scattering into the condensate, this effect may actually be negligible in a sufficiently dilute and low-temperature regime. 


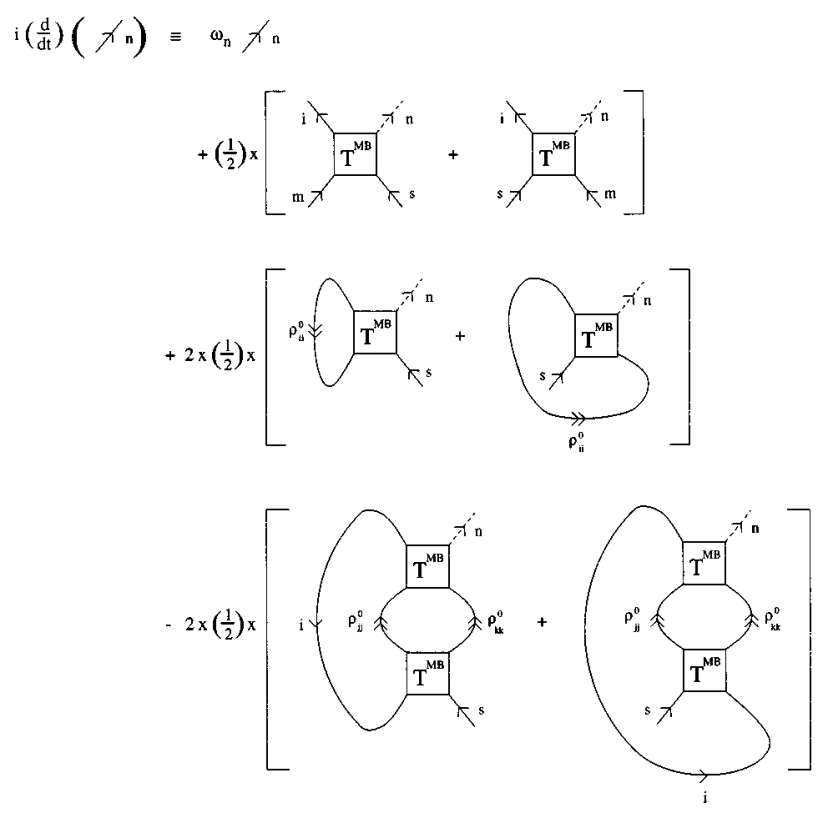

FIG. 3. This figure represents diagrammatically the evolution of the condensate mean value amplitude $z_{n}$ as given by Eq. (37). We have explicitly illustrated diagrams corresponding to direct and exchange terms, as well as the associated contribution prefactors. These factors have been suppressed in our mathematical analysis, by means of the definition (5) of a symmetrized matrix element. The factor $\rho^{0}$ indicates the population of excited states.

\section{EFFECT OF MEAN FIELD ON COLLISION DYNAMICS}

All discussion so far has been in terms of weakly interacting atoms, for which we can ignore the effect of the mean fields on the intermediate collisional states. In particular, in adiabatically eliminating the anomalous averages $\kappa_{j k}$ and $\lambda_{i j k}$, we have only considered certain of their contributions appearing in the equations of motion of Appendix A. These contributions correspond to those giving rise to $T$ matrices for both condensate-condensate and condensate-excitedstate interactions in the equation governing the evolution of the condensate mean field.

However, we know that the presence of mean fields (in terms of both condensate as well as excited states) will modify or "dress" the intermediate collisional states. In this section, we shall limit ourselves to the regime where all effects of the triplets can be ignored $(\lambda=\gamma=0)$. This will be shown to correspond to the HFB description of the system in terms of actual interatomic potentials, with the effect of mean fields during a collision generating the quasiparticle (Bogoliubov) dressing. In Sec. V we will discuss the validity of the simple GPE, as well as that of other conventional dilute Bose gas theories and we shall argue that a consistent theory necessitates an extension of the conventional meanfield theory (i.e., consideration of the triplets in our present language).

\section{A. The limit where $\lambda=\gamma=0$}

Let us initially recast the equations of motion of Appen$\operatorname{dix} \mathrm{A}$ in a simplified manner in the limit $\lambda=\gamma=0$. In terms of the anomalous correlation $\kappa_{j k}$, we note that Eq. (A6) contains the additional (dressing) contribution

$$
i \hbar \frac{d \kappa_{k j}}{d t}=\cdots+\sum_{s}\left[\eta_{k s} \kappa_{s j}+\kappa_{k s} \eta_{s j}^{*}\right]
$$

where $\eta$ is given by

$$
\eta_{k s}=2 \sum_{l t}\langle k l|\hat{V}| t s\rangle\left(z_{l}^{*} z_{t}+\rho_{t l}\right)
$$

From Eq. (A5), we also write down the equation of motion for $\rho_{j i}$. In Sec. III, we assumed $\kappa$ does not acquire a finite mean value between collisions (i.e., it relaxes to zero) and also ignored the off-diagonal $\rho$ elements. To deal with the conventional mean-field dressing, we shall now also allow for rapidly varying off-diagonal $\rho$ elements $\delta \rho_{j i}$, i.e., we shall substitute for $\rho_{j i}$ the expression [45]

$$
\rho_{j i}=\rho_{i i}^{0} \delta_{j i}+\delta \rho_{j i} .
$$

We shall further assume that $d \rho_{i i}^{0} / d t \simeq 0$. In these limits, we thus obtain the following set of equations for the rapid variations of $\delta \rho$ and $\delta \kappa$ during a binary collisional process:

$$
\begin{aligned}
& i \hbar \frac{d z_{n}}{d t}=\hbar \omega_{n} z_{n}+\sum_{i j k}\langle n i|\hat{V}| j k\rangle\left[z_{i}^{*} z_{j} z_{k}+2 \rho_{i i}^{0} \delta_{i j} z_{k}\right] \\
& +\sum_{i j k}\langle n i|\hat{V}| j k\rangle z_{i}^{*} \delta \kappa_{j k} \\
& +2 \sum_{i j k}\langle n i|\hat{V}| j k\rangle z_{k} \delta \rho_{j i} \\
& i \hbar \frac{d}{d t}\left(\delta \kappa_{k j}\right)=\hbar\left(\omega_{k}+\omega_{j}\right) \delta \kappa_{k j} \\
& +\sum_{s}\left[\eta_{k s} \delta \kappa_{s j}+\delta \kappa_{k s} \eta_{s j}^{*}\right] \\
& +\left(1+\rho_{k k}^{0}+\rho_{j j}^{0}\right) \Delta_{k j} \\
& +\sum_{s}\left[\delta \rho_{k s} \Delta_{s j}+\Delta_{k s} \delta \rho_{s j}^{*}\right] \\
& i \hbar \frac{d}{d t}\left(\delta \rho_{j i}\right)=\hbar\left(\omega_{j}-\omega_{i}\right) \delta \rho_{j i} \\
& +\sum_{r}\left[\eta_{j r} \delta \rho_{r i}-\delta \rho_{j r} \eta_{r i}\right] \\
& +\left(\rho_{i i}^{0}-\rho_{j j}^{0}\right) \eta_{j i} \\
& -\sum_{r}\left[\delta \kappa_{j r} \Delta_{r i}^{*}-\Delta_{j r} \delta \kappa_{r i}^{*}\right] .
\end{aligned}
$$

The physical significance of the various contributions appearing in these equations will be analyzed in detail below. First, however, we would like to point out that the limit $\lambda=\gamma=0$ of the equations of motion of Appendix A corresponds to the time-dependent Hartree-Fock-Bogoliubov 
equations, written in terms of actual interatomic potentials. These HFB equations can be alternatively generated by the following Hamiltonian [28]:

$$
H_{Q}=\frac{1}{2} \sum_{p q}\left\{h_{p q}\left(\hat{c}_{p}^{\dagger} \hat{c}_{q}+\hat{c}_{q} \hat{c}_{p}^{\dagger}\right)+\left(\Delta_{p q} \hat{c}_{p}^{\dagger} \hat{c}_{q}^{\dagger}+\Delta_{p q}^{*} \hat{c}_{q} \hat{c}_{p}\right)\right\}
$$

where

$$
\begin{aligned}
h_{p q}= & \langle p|\hat{h}| q\rangle=\langle p|\hat{\Xi}| q\rangle+\eta_{p q}=\langle p|\hat{\Xi}| q\rangle+2 \sum_{l t}\langle p l|\hat{V}| t q\rangle \\
& \times\left(z_{l}^{*} z_{t}+\rho_{t l}\right) .
\end{aligned}
$$

This operator takes account of all possible quadratic terms in bare particle fluctuation operators $\hat{c}_{i}$. In the literature, $H_{Q}$ is termed the quasiparticle Hamiltonian [28], as it describes fully an assembly of noninteracting quasiparticles. The dressing effect of the mean fields on the bare-particle states resulting from this Hamiltonian is conventionally referred to as quasiparticle dressing, and we shall now discuss its consequences.

\section{Quasiparticle dressing at zero temperature}

At $T \simeq 0$ there will be very few excited atoms in the assembly, so that we can approximate $\rho_{i i}^{0} \simeq 0$. We have already shown that the term $i \hbar\left(d \kappa_{k j} / d t\right) \propto \Delta_{k j}$ of Eq. (43) corresponds to the two-body $T$ matrix being generated in the interaction between condensed atoms. The terms containing $\eta$ in both $\delta \rho$ and $\delta \kappa$ [i.e., contributions (42) and (45)] give rise to the dressing of Fig. 4(a). This represents the simplest dressing term due to the mean field of the condensate in the intermediate collisional steps, and corresponds algebraically to shifting and mixing of the intermediate states and frequencies.

From Eqs. (44) and (47), we see that there further exist more complex contributions that depend on the coupling between $\delta \rho$ and $\delta \kappa$. We expect these to correspond to the anomalous quasiparticle (Bogoliubov) dressing, in much the same way creation and annihilation operators get coupled via the quasiparticle transformation

$$
\hat{c}_{i}=\sum_{l}\left[u_{i l} \hat{b}_{l}+v_{i l}^{*} \hat{b}_{l}^{\dagger}\right]
$$

where the $\hat{b}_{l}^{\dagger}$ correspond to quasiparticle annihilation (creation) operators.

Indeed, the combination of contributions (44) and (47) gives rise to intermediate dressing of the form of Fig. 4(c). We stress that the coupling between them implies that both diagrams in Fig. 4(c) are combined at any single intermediate step. To understand the form of the coupling, we have illustrated in Fig. 5 two typical diagrams in the evolution of the condensate mean field, along with their dressed equivalents due to the $\delta \rho-\delta \kappa$ coupling.

\section{Quasiparticle dressing at nonzero temperatures}

Observation of Eqs. (42) and (43) and (45) and (46) shows the following new features arising at $T>0$, where $\rho_{i i}^{0}$ becoms non-negligible due to thermally excited atoms. The
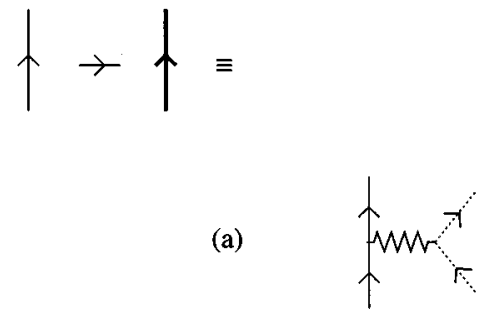

(b)

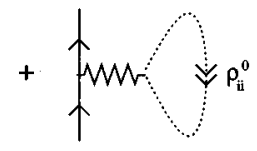

(c)

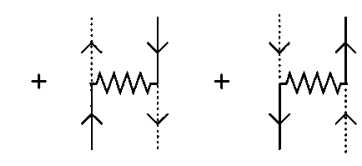

(d)

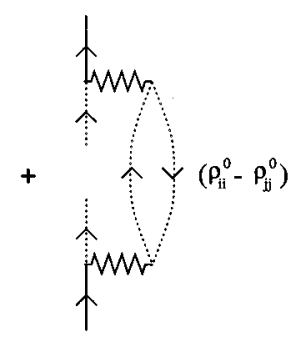

FIG. 4. This figure illustrates all different quasiparticle dressing effects on the unoccupied intermediate propagators of the two-body $T$ matrix [Fig. 2(a)]. Such dressing during an atomic collision arises due to the effect of (a) the condensate mean field, or (b) the mean field of thermally excited states. Additionally, the $\delta \rho-\delta \kappa$ coupling generates the anomalous terms of (c), corresponding to the creation, or annihilation of two condensed atoms, in favor of excited ones; these contributions do not appear separately, but are complementary of each other. (d) indicates the extra dressing due to the bubble diagrams $(46)$, which are associated with a factor $\left(\rho_{i i}^{0}-\rho_{j j}^{0}\right)$. We note that all above dressing effects could also be very straightforwardly included in the many-body loops of Figs. 2(b)-2(c).

first one, which we have already discussed quite extensively, is the replacement of the two-body $T$ matrix by the manybody one, due to enhanced condensate-condensate scattering via occupied excited states. Furthermore, the $\eta$ terms in Eqs. (42) and (45) also allow modifications in the intermediate collisional states due to the mean field of excited states [in analogy to the condensate mean field of Fig. 4(a)], as shown in Fig. 4(b).

The final modification we need to consider at finite temperatures is that due to the effect of the contribution (46). Adiabatic elimination of this contribution results in the generation of the so-called bubble diagrams shown in Fig. 4(d). Let us now give a brief physical explanation of the bubble diagrams. Consider the case of driving a trapped condensate by some external field. The condensate oscillations will induce transitions between excited states, and those will in turn act back on the condensate and modify its evolution. If the condensate is suitably perturbed, these terms will become 
(a)
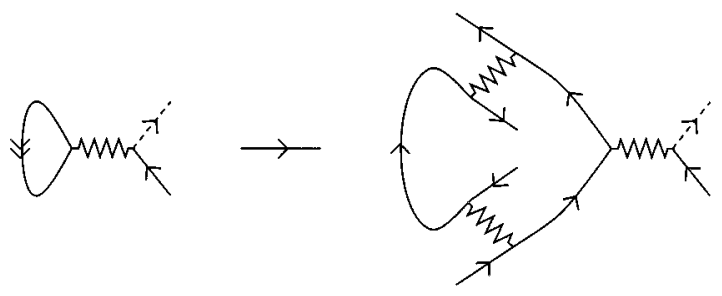

(b)

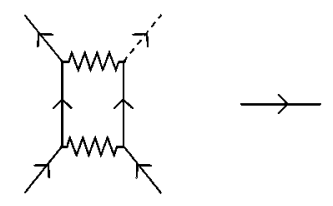

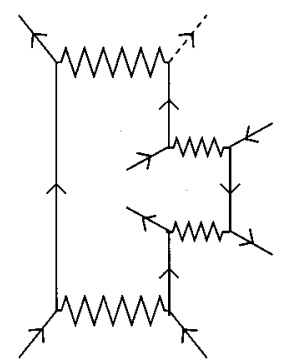

FIG. 5. This figure illustrates the cooperative action of the $\delta \rho$ $\delta \kappa$ coupling represented in Fig. 4(c), by means of two typical terms in the evolution of the condensate mean field, and their corresponding dressing due to this coupling: (a) shows the effect on the scattering between a condensed and an excited atom, whereas (b) corresponds to the dressing on a typical two-body ladder diagram.

dominant, thus implying that the many-body $T$ matrix is no longer the suitable parameter for the evolution of the condensate mean field. This would suggest that Eq. (37) is no longer valid, signaling a breakdown of the Markoffian approximation discussed in Secs. II and III. It is worth pointing out that although these bubble diagrams can usually be neglected in weakly interacting systems, they have recently been shown to be rather important near the critical temperature of the gas [46].

The interplay between all dressings of Figs. 4(a)-4(d) in the many-body $T$ matrix corresponds to what is conventionally referred to as the quasiparticle dressing for the interactions between condensed atoms. Indeed, the consistent combination of diagrams $4(\mathrm{a})-4(\mathrm{c})$ with the $T$-matrix elements will generate the well-known normal and anomalous selfenergies $\hbar \Sigma_{11}$ and $\hbar \Sigma_{12}$. However, we believe that the conventionally used finite-temperature mean-field theory does not actually include all these effects in a consistent fashion, as we shall argue below (Sec. V).

Following the above discussion on the dressing of interactions due to the presence of mean fields, we shall now turn our attention to the analysis of conventional Bose gas theories appearing in the literature.

\section{CONVENTIONAL THEORIES AND THEIR VALIDITY}

\section{A. The Gross-Pitaevskii equation}

In Sec. IV, we discussed the effect of mean fields on the intermediate collisional states in the limit $\lambda=\gamma=0$. This effect means we should use noninteracting quasiparticles, rather than bare particle states in our theoretical treatment. This dressing is not taken into account in the GPE, as was explicitly shown in its microscopic derivation in Sec. II.
However, this dressing can only be ignored in the limit of weak interactions, i.e., when $n U_{0} \ll \hbar \omega$. In order to derive the GPE, we also have to ignore the effects of the occupation of low-lying excitations during a collision (many-body effects). These restrictions give us the sufficient condition for the validity of the GPE, namely,

$$
n U_{0}, \quad k_{B} T \ll \hbar \omega,
$$

where $\hbar \omega$ is a typical energy separation between trap levels.

Effectively, the GPE only treats condensate-condensate scattering in vacuum (thus ignoring both quasiparticle dressing and many-body effects). In this limit, the $s$-wave scattering length indeed contains all relevant collisional information. It is conventionally argued that the corrections to the GPE due to the presence of mean field are purely diluteness corrections. If the conditions of Eq. (51) are fulfilled the corrections are indeed small and this statement is close to the truth. However, even this statement is open to critisism [39]. More generally, in the case of partial condensation $(T \neq 0)$, we maintain that the notion that diluteness is the issue is misleading.

\section{B. Zero-temperature Bogoliubov-de Gennes equations}

As mentioned above, the GPE takes no account of the finite $T=0$ depletion of the condensate, arising due to collisions between condensate atoms. This depletion is indeed very small in the case of typical experimentally studied condensates, as has been shown explicitly, e.g., by Hutchinson et al. [47]. One can, therefore, calculate the frequencies of the elementary excitations, to good accuracy, by finding normal modes of the linearized GPE, of the form

$$
\Phi(\mathbf{r}, t)=e^{-i \mu t}\left[\phi(\mathbf{r})+u(\mathbf{r}) e^{-i \omega t}+v^{*}(\mathbf{r}) e^{i \omega t}\right] .
$$

Here $\mu$ corresponds to the chemical potential of the undisturbed ground state and the condensate wave function has been represented by the condensate orbital $\phi(\mathbf{r})$. In addition, $\omega$ labels the frequency of the elementary excitations, whereas $u(\mathbf{r})$ and $v^{*}(\mathbf{r})$ correspond to the spatially dependent coefficients of the condensate's linear response to some driving field. This linear response approach has been discussed in $[6,7]$ where it has been shown that the substitution (52) is completely equivalent to carrying out the Bogoliubov transformation [48] on the fluctuating part of the Bose field operator $\hat{\Psi}-\langle\hat{\Psi}\rangle$, i.e., to the diagonalization of the binaryinteraction Hamiltonian for the assembly.

Thus, one obtains a set of three static coupled equations for $\phi(\mathbf{r}), u(\mathbf{r})$, and $v^{*}(\mathbf{r})$, known as the Bogoliubov-de Gennes (BdG) equations. These equations have been used to predict condensate shapes, densities, and the energies of elementary excitations at near zero temperatures. The results of the BdG equations appear to be in excellent agreement [8] with experiments as discussed in the general zerotemperature mean field theory review paper by Edwards et al. [7].

\section{The "essence" of Hartree-Fock-Bogoliubov}

We would like to point out that both use of Eq. (52) and the diagonalization discussed in [6,7] will only lead to the 
conventional $\mathrm{BdG}$ equations under the assumption $V(\mathbf{r}$ $\left.-\mathbf{r}^{\prime}\right)=U_{0} \delta\left(\mathbf{r}-\mathbf{r}^{\prime}\right)$. In this paper, we have argued that a $\delta$-function pseudopotential approximation can only be imposed on an "upgraded" effective interaction potential expressible in terms of the two-body $T$ matrix. This enormously complicates the discussion of the validity of certain approximate mean-field theories, and generates confusion as to precisely what set of equations is implied by the term "Hartree-Fock-Bogoliubov.",

We would argue that the HFB equations are, in fact, defined in terms of actual interatomic potentials. Thus, we define HFB as the set of coupled $z-\rho-\kappa$ equations of Appendix A (in which all interaction terms are left in terms of symmetrized matrix elements), in the limit $\lambda=\gamma=0$. The timedependent HFB equations defined in this way can thus be derived from the quasiparticle Hamiltonian (48) describing an assembly of noninteracting quasiparticles. We have already argued in Sec. III A, however, that such a theory cannot consistently describe the interaction between an atom in the condensate with an excited one. The rigorous way of doing this is by consideration of the $\lambda$ correlations. Bringing in the $\lambda$ correlations is equivalent to allowing the quasiparticles to interact with each other.

We shall now discuss what people conventionally refer to as the finite temperature mean field, or HFB approximation.

\section{Finite-temperature mean-field theory and the Popov approximation}

The mean-field treatment extensively discussed in the literature avoids dealing with actual interatomic potentials. Instead, one conventionally expresses the above finitetemperature HFB equations in terms of a $\delta$-function approximation. In the static case, these equations then take the form given below, which can be derived as a special case of our microscopic formalism. The equations are

$$
\begin{gathered}
\left\{-\frac{\hbar^{2}}{2 M} \nabla^{2}+V_{\text {trap }}(\mathbf{r})-\mu+U_{0}\left[N_{0}|\psi(\mathbf{r})|^{2}+2 \tilde{n}(\mathbf{r})\right]\right\} \psi(\mathbf{r}) \\
\quad+U_{0} \tilde{m}(\mathbf{r}) \psi^{*}(\mathbf{r})=0 \\
\hat{\mathcal{L}} u_{j}(\mathbf{r})+U_{0}\left[N_{0}\{\psi(\mathbf{r})\}^{2}+\tilde{m}(\mathbf{r})\right] v_{j}(\mathbf{r})=E_{j} u_{j}(\mathbf{r}), \\
\hat{\mathcal{L}} v_{j}(\mathbf{r})+U_{0}\left[N_{0}\left\{\psi^{*}(\mathbf{r})\right\}^{2}+\tilde{m}^{*}(\mathbf{r})\right] u_{j}(\mathbf{r})=-E_{j} v_{j}(\mathbf{r}),
\end{gathered}
$$

where we have defined the following quantities:

$$
\begin{gathered}
\tilde{n}(\mathbf{r})=\sum_{j}\left\{\left[\left|u_{j}(\mathbf{r})\right|^{2}+\left|v_{j}(\mathbf{r})\right|^{2}\right] N_{0}\left(E_{j}\right)+\left|v_{j}(\mathbf{r})\right|^{2}\right\}, \\
\tilde{m}(\mathbf{r})=\sum_{j} u_{j}(\mathbf{r}) v_{j}^{*}(\mathbf{r})\left[2 N_{0}\left(E_{j}\right)+1\right],
\end{gathered}
$$

and the operator

$$
\hat{\mathcal{L}}=-\frac{\hbar^{2}}{2 M} \nabla^{2}+V_{\text {trap }}(\mathbf{r})-\mu+2 U_{0}\left[N_{0}|\psi(\mathbf{r})|^{2}+\widetilde{n}(\mathbf{r})\right]
$$

Here, $N_{0}\left(E_{j}\right)=1 /\left(e^{\beta E_{j}}-1\right)$, and the above quantities are normalized in the usual manner [6]. These equations are being used at the moment to study the finite-temperature excitations of Bose-condensed gases $[47,49]$. Since these equations do not use actual interatomic potentials, we would rather not use the term HFB. In this paper, we shall call them the finite-temperature Bogoliubov-de Gennes equations, for want of a better name. The reason for making this distinction arises from our view that the approximations made in obtaining Eqs. (53)-(55) actually contain more physics than the conventional mean-field (HFB) approximation. This can be seen from our microscopic approach: In Sec. III, we have explicitly shown that the condensate-excited-state interactions can be considered in terms of an effective $T$-matrix interaction only once the triplet $\lambda$ is taken into account. We stress that this $T$-matrix effective interaction is implicit in the above form of the finite-temperature BdG equations (53)(55), since they have been written in terms of zero-range potentials. It is clearly important to bear in mind that, when one includes the $\lambda$ terms, one also brings in other effects that have been ignored in the coupled BdG equations, such as further dressing of intermediate collisional states. The finitetemperature BdG equations, therefore, have some inconsistencies built into them. How important these inconsistencies are in practice remains to be seen.

Some discussion has been recently focused around the so-called "Popov"' approximation of these equations, which corresponds to setting $\tilde{m}(\mathbf{r})=0$ in Eqs. (53)-(55) [24]. This approximation has been used to compute the finitetemperature excitations of a trapped Bose gas $[47,49]$. In these papers, the equations being solved are referred to as the coupled HFB-Popov equations. We shall use the labeling BdG-Popov, since even the Popov approximation of these equations goes beyond the conventional mean-field theory (due to the implicit inclusion of the $\lambda$ correlations). In our treatment, we have obtained these equations by adiabatically eliminating both anomalous averages $\kappa$ [or equivalently $\widetilde{m}(\mathbf{r})]$ and $\lambda[$ not explicitly present in Eqs. (53)-(55)]. Thus, the Popov limit appears to be one way of obtaining a consistent theory. Indeed, Griffin [24] has shown that such a theory is gapless. However, using Eqs. (53)-(55) as they stand, with a nonzero value of $\widetilde{m}(\mathbf{r})$, is clearly inconsistent, because we have shown that it is precisely the adiabatic elimination of $\widetilde{m}(\mathbf{r})$ that leads to the replacement of the interatomic potential by an effective interaction $U_{0}$.

Let us now discuss the validity of the Popov approximation. The BdG equations have been written in terms of the quasiparticle coherence factors $u_{j}(\mathbf{r})$ and $v_{j}(\mathbf{r})$. This shows explicitly that they include the effect of the condensate mean field on the initial and final collisional states, which modifies the bare particle states into noninteracting Bogoliubov quasiparticles. However, the analysis of Sec. IV shows that in this limit, we must also consider other effects, such as the dressing of intermediate collisional states and the occupation of these excitations. These many-body effects should be fully included in a consistent theory of condensate-condensate and condensate-excited-state collisions. The BdG-Popov theory does not take account of these effects, and we would thus expect it to deviate from the actual description of the system. Indeed, a basis-set simulation of excitation frequencies using the BdG-Popov equations [49] has revealed large (and quali- 
tative) differences from experimental data for temperatures above $0.6 T_{c}$. We are currently working on determining a more appropriate set of equations - similar to what we have termed finite-temperature $\mathrm{BdG}$ equations-that can be straightforwardly computed.

Our treatment shows that many-body effects are not the only effects that are not included in the BdG-Popov description, as discussed below.

\section{EFFECTS OF MEAN FIELDS BEYOND QUASIPARTICLE DRESSING}

The equations of Appendix A, indicate that the dressing discussed in Sec. IV is not the only effect the mean fields have during a collision. The dressing of Sec. IV arises from the quasiparticle Hamiltonian (48), which replaces the interacting (bare) atoms by non-interacting quasiparticles. However, we believe the theory should allow the quasiparticles to interact weakly with each other. These interactions will lead to more complex dressing of the intermediate collisional states. However, this is not the only reason for going beyond a quasiparticle description. We have extensively shown in this paper that, to obtain the evolution of the condensate mean field at finite temperatures, we need to consider the triplet $\lambda_{i j k}$ in Eq. (8). However, once including this, there appears to be no valid argument for neglecting the effects of the triplets in the remaining equations of Appendix A. For example, Eq. (A6) shows that when adiabatically eliminating $\kappa_{j k}$, we will also have to worry about triplet effects.

We thus believe the triplets have two effects. The reader is by now familiar with the first one, which is the necessity of triplets for deriving finite-temperature equations for condensate evolution. Secondly, to extend this argument even further, we also think that some of the triplets appearing in Eq. (A6) are actually needed in order to consistently combine the dressing diagrams of Fig. 4. We believe that a detailed study of the effects of the triplets may shed some light on why the dressed many-body $T$ matrix appears to go to zero as $T \rightarrow 0[19,46]$. This behavior implies a vanishing interaction (in the nonlinear Schrödinger equation) for collisions between two condensate atoms, which cannot be correct by itself, since we know that it is precisely these collisions that lead to the finite zero-temperature depletion of the condensate. We hope to discuss this, and related issues, in the future.

\section{CONCLUSIONS}

In this paper we have carried out an in-depth analysis of our microscopic description of the behavior of Bosecondensed systems at finite temperatures. The equations of [22] have been recast in Appendix A, for the convenience of the reader.

After a general introduction into our microscopic approach, we used the set of time-dependent Hartree-FockBogoliubov equations based on actual interatomic potentials (i.e., without making any assumptions about them), to derive a nonlinear Schrödinger equation (16) for the description of the condensate mean-field evolution at temperatures close to zero; in this limit, we can, to a good approximation, neglect the presence of excitations. We showed explicitly how to "upgrade" the interatomic interaction potential (5) to the bare-particle two-body $T$ matrix (18), which includes all repeated binary collisional processes in a trap in the absence of other particles. This is made possible by adiabatic elimination of the anomalous correlation $\langle c c\rangle$, which is not retained in the conventional derivation of the GPE.

Furthermore, we explicitly showed in what limits the bare-particle NLSE (16) reduces to the GPE (7), thus providing a clear microscopic derivation. We then generalized this NLSE to finite temperatures by including the effects of excited states. This results in a variety of new features: firstly, the occupation of excited states during a collision leads to the replacement of the two-body $T$ matrix for bare particles by the many-body one. Secondly, we must now also consider the effect of condensate-excited-state interactions in the evolution of the condensate mean field. In order to do this consistently, we have shown the necessity of extending the conventional mean-field theory by explicitly including the triplet $\left\langle c^{\dagger} c c\right\rangle$, which upgrades this interaction to the $T$ matrix. We have thus argued how to obtain a finite-temperature equation for the evolution of the condensate mean field, which includes kinetic contributions due to collisions between excited atoms. All such treatment is true in the limit of weak interactions, i.e., when $n U_{0} \ll \hbar \omega$. In this limit, it is reasonable to assume that the condensate mean field varies much more slowly than these anomalous averages, thus justifying the adiabatic elimination of anomalous averages and the free evolution of mean fields discussed in Secs. II and III.

When this condition does not hold, we must take account of the effect the mean fields have on the intermediate collisional states. This leads to dressing of the states accessed during a binary collision. The presence of such dressing contributions makes it extremely difficult to adiabatically eliminate the anomalous correlations $\langle c c\rangle$ and $\left\langle c^{\dagger} c c\right\rangle$ in favor of a consistent equation valid in all limits. In fact, we point out that the triplet $\left\langle c^{\dagger} c c\right\rangle$ must be rigorously dealt with in a consistent $T=0$ theory, due to the finite (albeit negligible) condensate depletion.

Our microscopic approach enables us to discuss the conventional mean-field theories currently used for describing the evolution of the condensate. In the first instance, we have argued that the Gross-Pitaevskii equation is strictly only valid in the regime $n U_{0}, k_{B} T \ll \hbar \omega$. Furthermore, we have argued that the conventionally used finite-temperature Bogoliubov-de Gennes equations [Eqs. (53)-(55)] contain some inconsistencies, which may be removed [24] if their treatment is restricted to the so-called Popov approximation [i.e., $\widetilde{m}(\mathbf{r})=0$ in Eqs. (53) $-(55)]$. In this case one speaks of the Popov approximation to BdG (or HFB-Popov). This naively suggests that the full BdG (HFB) theory is a better and more complete theory, which is clearly not the case when one is interested in the elementary excitations of a Bose condensate. To obtain a consistent theory, one must either consider BdG-Popov, or go beyond BdG (HFB) by including triplets to fix the gap in the spectrum of elementary excitations, as done in this paper. We therefore conclude that the terminology Popov approximation can be quite misleading, as it actually refers to a distinct set of equations to HFB. Furthermore, we have also argued that even the Popov limit of these equations may fail due to the neglect of many-body effects on the collisions in the gas. Indeed, recent simulations 
[48] have shown discrepancies with the experiments at temperatures beyond $0.6 T_{c}$. We believe this must be due to the back action of excited states onto the condensate mean field. In particular, we expect Eq. (37) to fail if the condensate is strongly driven in resonance with transitions between excited states, which will signal the onset of the importance of the bubble diagrams.

The analysis carried out in this paper shows precisely how hard it is to obtain a consistent mean-field theory for the description of partially Bose-condensed systems. An alternative description that may not face the same difficulties is based on Popov's approach of describing the homogeneous Bose gas in terms of an effective condensate density and phase. Such an approach has already been discussed by Ilinski and Stepanenko [50], and we hope more discussions in this area will appear in the future.

\section{ACKNOWLEDGMENTS}

The authors are grateful for helpful discussions with I. Cirac, M. Edwards, M. Bijlsma, S. Morgan, C. W. Clark, R. J. Dodd, and P. Julienne. N.P.P. would like to thank the Alexander S. Onassis Public Benefit Foundation (Greece) for financial support, whereas K.B. would like to acknowledge funding from the European Community, under the TMR network Program No. FMRX-CT96-0002.

\section{APPENDIX A: GENERALIZED MEAN-FIELD EQUATIONS}

In this appendix we give the full set of self-consistent mean-field equations derived in [22], subject to a decoupling approximation of correlations of four and five fluctuation operators. We have reexpressed these equations in a form that allows us to identify the physical importance of all the individual contributions. We remind the reader of the following definitions

$$
\begin{gathered}
\rho_{j i}=\left\langle\hat{c}_{i}^{\dagger} \hat{c}_{j}\right\rangle, \quad \kappa_{j k}=\left\langle\hat{c}_{k} \hat{c}_{j}\right\rangle, \quad \lambda_{i j k}=\left\langle\hat{c}_{i}^{\dagger} \hat{c}_{j} \hat{c}_{k}\right\rangle, \\
\gamma_{i j k}=\left\langle\hat{c}_{i} \hat{c}_{j} \hat{c}_{k}\right\rangle, \\
\eta_{j r}=2 \sum_{l t}\langle j l|\hat{V}| t r\rangle\left(z_{l}^{*} z_{t}+\rho_{t l}\right), \\
\Delta_{k j}=\sum_{m s}\langle k j|\hat{V}| m s\rangle\left[z_{m} z_{s}+\kappa_{m s}\right] .
\end{gathered}
$$

In writing down the equations of motion, we have assumed we are working in a bare particle basis, where the operator $\hat{\Xi}=-\left(\hbar^{2} \nabla^{2}\right) /(2 m)+V_{\text {trap }}(\mathbf{r})$ is diagonal, i.e., $\langle k|\hat{\hat{\Xi}}| n\rangle$ $=\hbar \omega_{n} \delta_{n k}$. Furthermore, the equations given below are expressed in terms of the actual interatomic potential experienced between two atoms at each collisional vertex. We thus obtain the following set of equations:

$$
\begin{aligned}
& i \hbar \frac{d z_{n}}{d t}=\hbar \omega_{n} z_{n}+\sum_{i j k}\langle n i|\hat{V}| j k\rangle\left[z_{i}^{*} z_{j} z_{k}+\kappa_{j k} z_{i}^{*}+2 \rho_{j i} z_{k}+\lambda_{i j k}\right] \\
& i \hbar \frac{d \rho_{j i}}{d t}=\hbar\left(\omega_{j}-\omega_{i}\right) \rho_{j i}+\sum_{r}\left[\eta_{j r} \rho_{r i}-\rho_{j r} \eta_{r i}\right]-\sum_{r}\left[\kappa_{j r} \Delta_{r i}^{*}-\Delta_{j r} \kappa_{r i}^{*}\right]+\sum_{r m s}\langle j r|\hat{V}| m s\rangle\left(2 \lambda_{m r i}^{*} z_{s}+\lambda_{i m s} z_{r}^{*}\right) \\
& -\sum_{r m s}\langle m s|\hat{V}| r i\rangle\left(2 \lambda_{m r j} z_{s}^{*}+\lambda_{j m s}^{*} z_{r}\right) \\
& i \hbar \frac{d \kappa_{k j}}{d t}=\hbar\left(\omega_{k}+\omega_{j}\right) \kappa_{k j}+\Delta_{k j}+\sum_{s}\left[\rho_{k s} \Delta_{s j}+\Delta_{k s} \rho_{s j}^{*}\right]+\sum_{s}\left[\eta_{k s} \kappa_{s j}+\kappa_{k s} \eta_{s j}^{*}\right]+\sum_{r m s}\left\{\langle k r|\hat{V}| m s\rangle\left[2 \lambda_{r s j} z_{m}+\gamma_{m s j} z_{r}^{*}\right]+(k \leftrightarrow j)\right\} \\
& i \hbar \frac{d}{d t}\left(\lambda_{i j k}\right)=\hbar\left(\omega_{k}+\omega_{j}-\omega_{i}\right) \lambda_{i j k}+\sum_{m s}\langle j k|\hat{V}| m s\rangle\left(2 \rho_{m i} z_{s}+\lambda_{i m s}\right)+\sum_{r m s}\left\{\langle k r|\hat{V}| m s\rangle \rho_{j r}\left[2 \rho_{m i} z_{s}+\lambda_{i m s}\right]+(k \leftrightarrow j)\right\} \\
& -\sum_{r m s}\left\{\langle m s|\hat{V}| i r\rangle \rho_{j m} \rho_{k s} z_{r}+(k \leftrightarrow j)\right\}+\sum_{s}\left\{\lambda_{i j s} \eta_{s k}^{*}+\lambda_{j i s}^{*} \Delta_{s k}+(k \leftrightarrow j)\right\}-\sum_{s}\left[\eta_{i s}^{*} \lambda_{s j k}-\Delta_{j s}^{*} \gamma_{s i k}\right] \\
& +\sum_{r m s}\left\{\langle k r|\hat{V}| m s\rangle 2 \rho_{m i}\left(\kappa_{j s} z_{r}^{*}+\lambda_{r j s}\right)+(k \leftrightarrow j)\right\}-\sum_{r m s}\langle m s|\hat{V}| i r\rangle\left\{\kappa_{r k}\left(2 \rho_{j m} z_{s}^{*}+\lambda_{j m s}^{*}\right)+(k \leftrightarrow j)\right\} \\
& +\sum_{r m s}\left\{\langle k r|\hat{V}| m s\rangle\left[2 \kappa_{m j}\left(\kappa_{i r}^{*} z_{s}+\lambda_{s i r}^{*}\right)+\kappa_{i r}^{*} \gamma_{s m j}\right]+(k \leftrightarrow j)\right\}-\sum_{r m s}\langle m s|\hat{V}| i r\rangle\left\{2 \rho_{j m} \lambda_{s k r}+(k \leftrightarrow j)\right\}, \\
& i \hbar \frac{d}{d t}\left(\gamma_{i j k}\right)=\hbar\left(\omega_{i}+\omega_{j}+\omega_{k}\right) \gamma_{i j k}+\sum_{m s}\langle i j|\hat{V}| m s\rangle\left(2 \kappa_{k m} z_{s}+\gamma_{k m s}\right)+\sum_{r m s}\left\{\langle i r|\hat{V}| m s\rangle \rho_{j r}\left[2 \kappa_{k m} z_{s}+\gamma_{k m s}\right]+(i \leftrightarrow j)\right\} \\
& +\sum_{r m s}\langle i r|\hat{V}| m s\rangle\left\{\kappa_{m k}\left[\kappa_{j s} z_{r}^{*}+2 \lambda_{r j s}\right]+(k \leftrightarrow j)\right\}+\sum_{s} \eta_{i s} \gamma_{s j k}+(i \leftrightarrow j \leftrightarrow k)
\end{aligned}
$$


$i\left(\frac{d}{d t}\right)\left(x_{n}\right) \equiv \omega_{n} x_{n}$

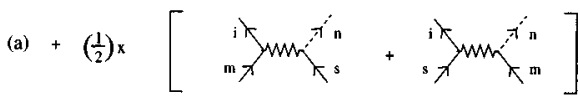

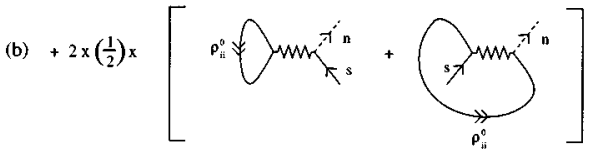

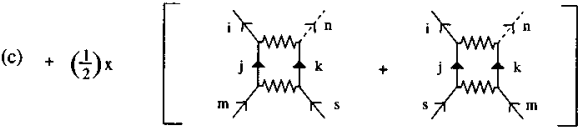

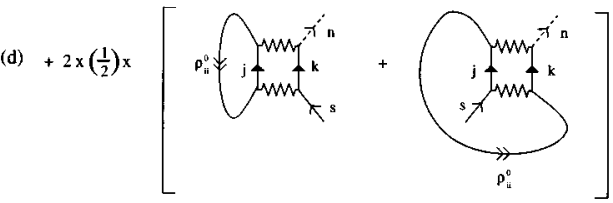

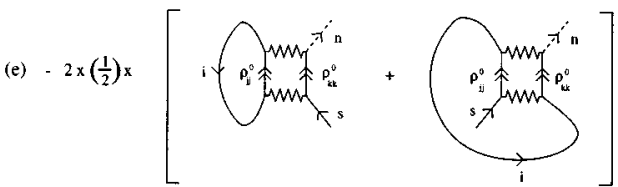

FIG. 6. This figure illustrates the evolution of the condensate mean field to second order in the interaction potential. (a)-(d) show the manybody $T$-matrix terms in the interactions (to order $V^{2}$ ) between two condensed atoms [(a),(c)], or one condensed atom with an excited one $[(\mathrm{b}),(\mathrm{d})]$. (e) shows the contribution to condensate evolution, due to the interaction of two excited atoms. (f) corresponds to the bubble diagrams, which have a factor $\left(\rho_{i i}^{0}-\rho_{j j}^{0}\right)$ associated with them (indicated by the open arrows). Continuous lines with one "free" end point have a factor $z$ associated with them, and the thick arrows due to excited-state occupation have been defined in Fig. 2(c).

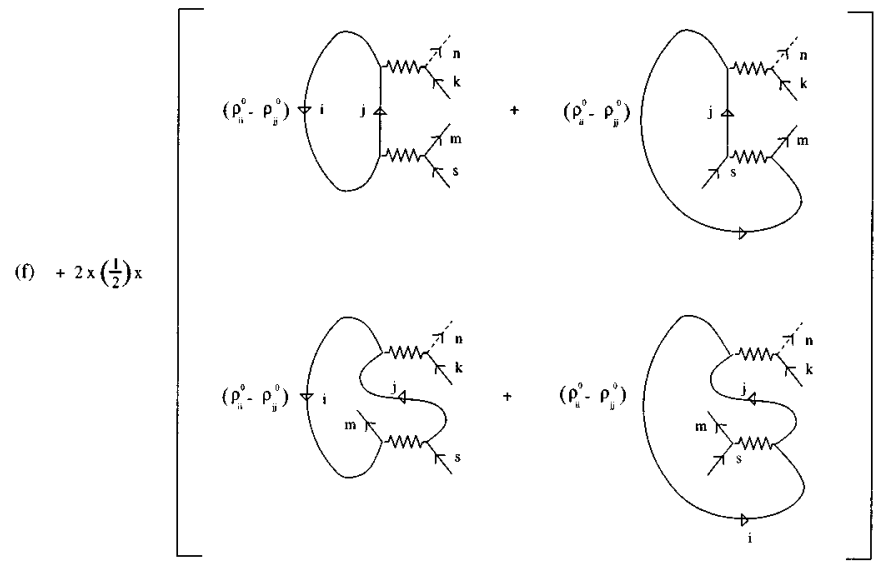

[The term $+(i \leftrightarrow j \leftrightarrow k)$ in the last equation indicates summation of all terms appearing in Eq. (A8), under cyclic rotation of the indices $i, j$, and $k$-except, of course, the first "free evolution" contribution.]

\section{APPENDIX B: CONDENSATE EVOLUTION TO SECOND ORDER IN THE INTERACTION POTENTIAL}

In this appendix we give a systematic categorization of all contributions to the condensate mean-field evolution, to second order in the interaction potential $V$. We shall carry this out by making use of the decomposition (40) of the single-particle correlation $\rho$ into a slowly varying diagonal $\left(\rho^{0}\right)$ and a rapidly evolving off-diagonal element $(\delta \rho)$. The implicit assumption made here is that the atoms are weakly interacting, so that the bare particle basis may be used, to a good approximation, for the description of the system. Furthermore, we have already argued in Sec. III C that the equations of the Appendix do not take account of the evolution of excited states due to quantum Boltzmann-type effects, which can be included by suitable treatment of the $\left\langle c^{\dagger} c^{\dagger} c c\right\rangle$ correlations. We shall therefore assume that during a collision $d \rho_{i i}^{0} / d t \simeq 0$, which gives for the condensate mean-field evolution to $V^{2}$, the expression 


$$
\begin{aligned}
i \hbar \frac{d z_{n}}{d t}= & \hbar \omega_{n} z_{n}+\sum_{i m s}\langle n i|\hat{V}| m s\rangle z_{i}^{*} z_{m} z_{s} \\
& +2 \sum_{i s}\langle n i|\hat{V}| i s\rangle \rho_{i i}^{0} z_{s} \\
& +\sum_{i j k}\langle n i|\hat{V}| j k\rangle \sum_{m s} \int \frac{d t^{\prime}}{i \hbar} e^{-i\left(\omega_{k}+\omega_{j}-\omega_{m}-\omega_{s}\right)\left(t-t^{\prime}\right)}\left[1+\rho_{j j}^{0}\left(t^{\prime}\right)+\rho_{k k}^{0}\left(t^{\prime}\right)\right]\langle j k|\hat{V}| m s\rangle z_{i}^{*}(t) z_{m}(t) z_{s}(t) \\
& +\sum_{i j k}\langle n i|\hat{V}| j k\rangle \sum_{s} \int \frac{d t^{\prime}}{i \hbar} e^{-i\left(\omega_{k}+\omega_{j}-\omega_{i}-\omega_{s}\right)\left(t-t^{\prime}\right)}\left[1+\rho_{j j}^{0}\left(t^{\prime}\right)+\rho_{k k}^{0}\left(t^{\prime}\right)\right]\langle j k|\hat{V}| i s\rangle 2 \rho_{i i}^{0}(t) z_{s}(t) \\
& -\sum_{i j k}\langle n i|\hat{V}| j k\rangle \sum_{s} \int \frac{d t^{\prime}}{i \hbar} e^{-i\left(\omega_{k}+\omega_{j}-\omega_{i}-\omega_{s}\right)\left(t-t^{\prime}\right)} 2 \rho_{j j}^{0}\left(t^{\prime}\right) \rho_{k k}^{0}\left(t^{\prime}\right)\langle j k|\hat{V}| i s\rangle z_{s}(t) \\
& +4 \sum_{i j k}\langle n i|\hat{V}| j k\rangle z_{k}(t) \sum_{m s} \int \frac{d t^{\prime}}{i \hbar} e^{-i\left(\omega_{i}+\omega_{m}-\omega_{j}-\omega_{s}\right)\left(t-t^{\prime}\right)}\left[\rho_{i i}^{0}\left(t^{\prime}\right)-\rho_{j j}^{0}\left(t^{\prime}\right)\right]\langle j m|\hat{V}| i s\rangle\left[z_{m}^{*}(t) z_{s}(t)+\rho_{s s}^{0}(t) \delta_{s m}\right],
\end{aligned}
$$

where $t^{\prime}$ acquires values within the range $-\infty$ to $t$. This equation has been diagrammatically depicted in Fig. 6, where we also give the contribution factors of each diagram.

The terms in Eqs. (B1) and (B2) represent the free evolution of the condensed particle, and the interaction of one condensed atom, either with another condensed atom [Fig. 6(a)], or with an excited one [Fig 6(b)]. Equations (B3) and (B4) give the lowest-order corrections of the actual interatomic potential, due to its replacement by the many-body $T$ matrix, in both cases of condensate-condensate [Fig. 6(c)] and condensate-excited-state interactions [Fig. 6(d)]. The contribution (B5) - Fig. 6(e) — arises due to the interactions between two excited atoms and ensures the correct scattering amplitude factors for condensate evolution, as already explained in Sec. III C. Finally, Eq. (B6) corresponds to the bubble diagrams of the many-body formalism, which are shown in Fig. 6(f).

At this point, we should really comment on the consistency of the above equation for condensate evolution. In obtaining this equation, we have not dealt with all quantities in the same manner. A fully consistent treatment would be to assign a slowly varying and a rapidly evolving part to each of the quantities $\rho, z$, and $\kappa$ (which would also generate two parts for both $h$ and $\Delta$ ), as well as the triplets $\lambda$ and $\gamma$ and then deal with them as a closed system. We shall return to this issue in a forthcoming presentation.
[1] M. H. Anderson, J. R. Enscher, M. R. Matthews, C. E. Wieman, and E. A. Cornell, Science 269, 198 (1995).

[2] K. B. Davis, M.-O. Mewes, M. R. Andrews, N. J. van Druten, D. S. Durfee, D. M. Kurn, and W. Ketterle, Phys. Rev. Lett. 75, 3969 (1995).

[3] C. C. Bradley, C. A. Sackett, J. J. Tollett, and R. G. Hulet, Phys. Rev. Lett. 75, 1687 (1995); C. C. Bradley, C. A. Sackett, and R. G. Hulet, ibid. 78, 985 (1997).

[4] E. P. Gross, J. Math. Phys. 4, 195 (1963); L. Pitaevskii, Sov. Phys. JETP 13, 451 (1961).

[5] M. Edwards and K. Burnett, Phys. Rev. A 51, 1382 (1995); P. A. Ruprecht, M. J. Holland, K. Burnett, and M. Edwards, ibid. 51, 4704 (1995); M. Edwards, R. J. Dodd, C. W. Clark, P. A. Ruprecht, and K. Burnett, ibid. 53, 1950 (1996); R. J. Dodd, M. Edwards, C. J. Williams, C. W. Clark, M. J. Holland, P. A. Ruprecht, and K. Burnett, ibid. 54, 661 (1996); R. J. Dodd, J. Res. Natl. Inst. Stand. Technol. 101, 545 (1996).

[6] P. A. Ruprecht, M. Edwards, K. Burnett, and C. W. Clark, Phys. Rev. A 54, 4178 (1996).

[7] M. Edwards, R. J. Dodd, C. W. Clark, and K. Burnett, J. Res.
Natl. Inst. Stand. Technol. 101, 553 (1996).

[8] M. Edwards, P. A. Ruprecht, K. Burnett, R. J. Dodd, and C. W. Clark, Phys. Rev. Lett. 77, 1671 (1996).

[9] M. J. Holland and J. Cooper, Phys. Rev. A 53, R1954 (1996).

[10] G. Baym and C. Pethick, Phys. Rev. Lett. 76, 6 (1996).

[11] A. L. Fetter, Phys. Rev. A 53, 4245 (1996).

[12] F. Dalfovo and S. Stringari, Phys. Rev. A 53, 2477 (1996); F. Dalfovo, L. Pitaevskii, and S. Stringari, J. Res. Natl. Inst. Stand. Technol. 101, 537 (1996).

[13] D. S. Jin, J. R. Ensher, M. R. Matthews, C. E. Wieman, and E. Cornell, Phys. Rev. Lett. 77, 420 (1996); M.-O. Mewes, M. R. Andrews, N. J. van Druten, D. M. Murn, D. S. Durfee, C. G. Townsend, and W. Ketterle, ibid. 77, 988 (1996); D. S. Jin, M. R. Matthews, J. R. Ensher, C. E. Wieman, and E. A. Cornell, ibid. 78, 764 (1997).

[14] When referring to weak interactions in this paper, we mean that the effect of the medium on a typical (repeated) binary collision is not large enough to cause appreciable modification of the effective interaction from the two-body $T$ matrix $\left(T^{2 \mathrm{~B}}\right)$. This is indeed true if $n T^{2 \mathrm{~B}} \ll \hbar \omega$, where $n$ is the atomic density 
and $\hbar \omega$ the trap level spacing.

[15] S. T. Beliaev, Zh. Éksp. Teor. Fiz. 34, 433 (1958) [ Sov. Phys. JETP 7, 299 (1958)].

[16] V. N. Popov, Functional Integrals in Quantum Field Theory and Statistical Physics (Reidel, Dordrecht, 1983).

[17] K. Huang and C. N. Yang, Phys. Rev. 105, 767 (1957).

[18] T. D. Lee, K. Huang, and C. N. Yang, Phys. Rev. 106, 1135 (1957).

[19] M. Bijlsma and H. T. C. Stoof, Phys. Rev. A 55, 498 (1997).

[20] H. Shi, Ph.D. thesis, University of Toronto (1997).

[21] H. T. C. Stoof, Phys. Rev. A 45, 8398 (1992).

[22] N. P. Proukakis and K. Burnett, J. Res. Natl. Inst. Stand. Technol. 101, 457 (1996).

[23] An alternative approach using Green's functions has been independently carried out by Griffin [24]. In Griffin's approach, however, the HFB equations are considered in the limit of $\delta$-function pseudopotentials, as will be discussed in more detail in Sec. V.

[24] A. Griffin, Phys. Rev. B 53, 9341 (1996).

[25] D. A. Huse and E. D. Siggia, J. Low Temp. Phys. 46, 137 (1982).

[26] P. Dörre, H. Haug, and D. B. Tran Thoai, J. Low Temp. Phys. 35, 465 (1979).

[27] D. H. Kobe, Ann. Phys. (N.Y.) 47, 15 (1968).

[28] J. P. Blaizot and G. Ripka, Quantum Theory of Finite Systems (MIT Press, Cambridge, MA, 1986).

[29] H. T. C. Stoof, Phys. Rev. A 49, 3824 (1994).

[30] F. Lalöe, in Bose-Einstein Condensation, edited by A. Griffin, D. W. Snoke and S. Stringari (Cambridge University Press, Cambridge, MA, 1995).

[31] N. P. Proukakis and K. Burnett, Philos. Trans. R. Soc. London, Ser. A (to be published).

[32] We point out that this distinction between the matrix elements $\langle n i|\hat{V}| j k\rangle$ and $(n i|\hat{V}| j k)$ was not explicitly analyzed in [22], although all equations of motion appearing there [i.e., all equations after (17) in [22]] made use of this symmetrized matrix element of the interaction potential. We note that the use of the symmetrized matrix element greatly simplifies all subsequent expressions, and has therefore also been adopted in this paper.
[33] E. Fermi, Riverca Sci. 7, 13 (1936).

[34] W. Glöckle, The Quantum Mechanical Few-Body Problem (Springer, Berlin, 1983).

[35] P. Nozières and D. Pines, The Theory of Quantum Liquids, Volume II (Addison-Wesley, New York, 1990).

[36] K. Huang and P. Tommasini, J. Res. Natl. Inst. Stand. Technol. 101, 435 (1996).

[37] K. Huang, Statistical Mechanics, 2nd ed. (John Wiley and Sons, New York, 1987).

[38] M. Lewenstein and L. You, Phys. Rev. A 53, 909 (1996).

[39] N. P. Proukakis, D. Phil. thesis, University of Oxford (1997).

[40] H. T. C. Stoof, M. Bijlsma, and M. Houbiers, J. Res. Natl. Inst. Stand Technol. 101, 443 (1996).

[41] Here we are assuming that the anomalous correlation $\lambda_{i j k}$ acquires no equilibrium value in the assembly, just as was done in Sec. II for $\kappa_{j k}$.

[42] H. T. C. Stoof, Phys. Rev. Lett. 78, 768 (1997).

[43] We would like to point out that kinetic processes have also been discussed in C. W. Gardiner and P. Zoller, Phys. Rev. A 55, 2902 (1997).

[44] This was first suggested to us in terms of our formalism by Ignacio Cirac.

[45] At this point, we note that we are interested in the evolution of the condensate mean field. If we were dealing with dressing on the evolution of excited bare particle states, for example, we would also need to consider a split $z \rightarrow z^{0}+\delta z$; a fully selfconsistent approach would further necessitate $\kappa \rightarrow \kappa^{0}+\delta \kappa[\mathrm{im}-$ plying that we no longer need to assume that $\kappa\left(t_{0}\right) \rightarrow 0$ as $t_{0}$ $\rightarrow-\infty]$, as well as $\eta \rightarrow \eta^{0}+\delta \eta$ and $\Delta \rightarrow \Delta^{0}+\delta \Delta$. We hope to return to this exact linearization procedure in a future publication dealing with the damping of quasiparticles.

[46] M. Bijlsma and H. T. C. Stoof, Phys. Rev. A 54, 5085 (1996).

[47] D. A. W. Hutchinson, E. Zaremba, and A. Griffin, Phys. Rev. Lett. 78, 1842 (1997).

[48] N. N. Bogoliubov, J. Phys. (Moscow) 11, 23 (1947).

[49] R. J. Dodd, M. Edwards, C. W. Clark, and K. Burnett, Phys. Rev. A 57, 32 (1998).

[50] K. N. Ilinski and A. S. Stepanenko, e-print cond-mat/9607202, 1997. 